\title{
Trazando líneas: estimación de la canasta básica de alimentos y líneas de pobreza en Bolivia para el periodo 1999-2012
}

Drawing the line: Estimating the basic food basket and poverty lines in Bolivia during the 1999 - 2012 period

Gary Mena* Wilson Jiménez**

\section{Resumen:}

La construcción de la línea de pobreza es el paso más crítico y difícil en la medición de la pobreza, pues establece el umbral que define quién es o no es pobre. En el presente documento se establecen los criterios y se utiliza la información disponible para construir Canastas Básicas Alimentarias a partir de la Encuesta Continua de Hogares 2003-2004, y se obtiene una valoración de dichas canastas con los precios del IPC base 2007. A partir de las Canastas Básicas Alimentarias se estimaron las líneas de pobreza extrema y moderada para las ciudades de Santa Cruz, La Paz y Cochabamba, así como para el resto urbano y el área rural de Bolivia para el periodo 1999-2012. Las actuales estimaciones de pobreza monetaria en Bolivia aún tienen como referencia la canasta básica de alimentos construida en 1995, bajo un contexto de mayor restricción y escasez de información acerca de patrones de consumo, una estructura demográfica de hace dos décadas y criterios desactualizados sobre las necesidades nutricionales.

Palabras clave: Líneas de pobreza, canasta básica de alimentos, precios.

\footnotetext{
Fundación ARU. Contacto: gmena@aru.org.bo

* Fundación ARU. Contacto: wjimenez@gmail.com
} 


\section{Abstract:}

To establish poverty line(s) is the most critical and hardest step in the measurement of poverty because it defines who is and is not poor. Bolivias official estimates are based on an outdated household survey which was constrained to the urban area only. In this paper we outline the assumptions made to construct basic food baskets for 5 domains and present results from the 2003-2004 expenditure income household survey based on the food energy intake methodology. Then we update the results for the 1999-2012 period using the prices from the Consumer Price Index survey and show the significant differences between the estimations for the urban and rural area as well as the differences with the international poverty line that is the reference for international poverty comparisons.

Key words: Poverty lines, basic food basket, prices.

\section{Clasificación/Classification JEL: D12,I32}

\section{Introducción ${ }^{1}$}

La construcción de la línea de pobreza es el paso más crítico y difícil en la medición de la pobreza, pues establece el umbral que define quién es o no es pobre. La dificultad se debe en parte a que existen diferentes metodologías para la estimación de líneas de pobreza, siendo tres los enfoques más conocidos: (i) Costo de necesidades básicas (CNB), (ii) ingesta de calorías (FEI, por sus siglas en inglés) y (iii) líneas de pobreza relativas. Otra alternativa incluye laestimación de la pobreza en función ala línea de pobreza internacional establecida por los expertos del Banco Mundial.

Los dos primeros métodos asocian las necesidades básicas a los requerimientos energéticos, lo que no es una tarea trivial, ya que los requerimientos varían entre los individuos y a lo largo del tiempo para un determinado individuo en función a los niveles de actividad, entre otras cosas. Ambos métodos pueden brindar resultados sumamente distintos incluso para un mismo país a partir de los mismos datos (Ravallion et al.,1994). El tercer método establece la línea de pobreza en relación a algún parámetro, i.e, la mediana del ingreso per cápita.

1 Los puntos de vista y opiniones expresados en este documento son de los autores y no reflejan necesariamente los puntos de vista y opiniones de la Fundación ARU o cualquier otra institución a la que estén afiliados. Esta versión fue beneficiada por los comentarios de Tito Velasco en el Taller de Investigación Aplicada (ARU-IISEC, Junio 2013). 
Es necesario reconocer que todos los métodos tienen sus ventajas y desventajas, las cuales han sido estudiadas en los trabajos, por ejemplo, de Deaton (2001), Ravallion (1998, 2010) o Ravallion y Bidani (1994).

Las líneas de pobreza estimadas a partir de método de la FEI y sus variantes fueron aplicadas en los trabajos de Dandekar y Rath (1971), Greer y Thorbecke (1986), Osmani (1982), Paul (1989), así como por distintos gobiernos de países de ingresos bajos. A pesar de la simplicidad de los datos y métodos requeridos, el problema principal es que las líneas resultantes no son necesariamente consistentes en relación a la teoría utilitarista o a la de capacidades (Ravallion, 2010).

El método del $\mathrm{CNB}$ es uno de los más ampliamente usados para calcular líneas de pobreza en países de ingresos no altos. Debido mayormente a las limitaciones de los datos disponibles, las necesidades básicas se limitan usualmente a las necesidades energéticas / nutricionales, dando lugar a la línea de pobreza extrema. Es posible derivar la parte no alimentaria de las necesidades básicas a través de métodos complementarios (véase Ravallion, 1998), y obtener la línea de pobreza moderada. En este casola parte no alimentaria es la que se encuentra vulnerable a las críticas que se hacen al método de la FEI.

Dado que el Costo de Necesidades Básicas es un método normativo, al igual que los demás, el hecho de que permita relacionar la línea de pobreza a requerimientos energéticos y los patrones de consumo de un determinado sub-grupo de la población permite a esta metodología no estar sujeta a criterios arbitrarios. Sin embargo, se debe reconocer que este método también esta sujeto a diversas críticas en función a los datos disponibles.

Algunos estudios previos al respecto de la CBA en Bolivia son: el de Morales (1983), quien establece una canasta mínima vital para medir el poder de compra de los salarios en periodos de alta inflación; los de UDAPSO (1993 y 1995), este último en coordinación con CEPAL, que establecen la CBA para la medición de la pobreza monetaria en Bolivia. Para el área rural, UDAPE estimó las líneas de pobreza a partir de los datos de la Encuesta de Evaluación de Impacto del Fondo de Inversión Social (FIS).

El presente documento tiene el propósito de actualizar los criterios usados en la construcción de canastas básicas alimentarias, pues son el sustento de las líneas de pobreza en Bolivia. La revisión de las líneas de pobreza nacionales se justifica dado que la actual 
medición publicada regularmente por las entidades del Estado tiene bases desactualizadas. Por ejemplo:

Utilizan una versión de 1985 para el cálculo de requerimientos energéticos de la población, que ya fue actualizada en 2003 por organismos internacionales.

- Las necesidades medias de energía corresponden a las características sociodemográficas de 1995.

- La estructura del consumo alimentario proviene de la Encuesta de Presupuestos Familiares de 1991, la cual tenía representatividad únicamente para cuatro ciudades y no así para el área rural.

- Para el área rural se usó información de una encuesta sumamente limitada para generar estructuras de consumo.

- Los precios utilizados corresponden a la especificación del IPC con base en 1991 y no tienen una metodología de enlace con el actual IPC base 2007.

- La última estimación de las líneas de pobreza es para 2009.

En función a la información disponible, este trabajo trata de estimar la CBApara cinco dominios específicos: (i) La Paz, (ii) Cochabamba, (iii) Santa Cruz, (iv) Resto urbano y (v) Área rural; siguiendo inicialmente la metodología de la CEPAL. Una vez establecida la CBA, se procede a estimar las líneas de pobreza para los dominios anteriormentedefinidos.

La estructura del documento es de la siguiente manera: en la sección 2 se hace una descripción de las fuentes de información. Los cálculos de la CBA se documentan en la sección 3. Luego se procede al cálculo de las líneas de indigencia y pobreza en las secciones 5 y 6. Finalmente, se desarrollan algunas consideraciones finales y las implicaciones de política en la sección 7 .

\section{Fuentes de información y datos}

La principal fuente de información para la construcción de las CBA fue la Encuesta Continua de Hogares realizada entre noviembre de 2003 y noviembre de 2004. Dicha encuesta se aplicó a los hogares con una distribución temporal de 27 decenas ${ }^{2}$ (períodos de

2 En los tres primeros días de visita al hogar se recolectaron datos sobre las características del hogar, empleo e ingresos, mientras que en los siguientes siete días se llenaron las libretas de gastos diarios del hogar y gastos 
10 días) de levantamiento de información, con una cobertura nacionaly representatividad por área, departamentos y las nueve ciudades capitales de departamento (INE, 2005).

La ECH recolectó información de gastos e ingresos de aproximadamente 9000 hogares, seleccionados bajo una estrategia demuestreo estratificadosegúnel tamaño delaslocalidades y una subestratificación con el índice de Necesidades Básicas Insatisfechas (NBI) calculada en 2002 con datos del Censo Nacional de Población y Vivienda de 2001 (Censo 2001). Las variables que corresponden al gasto familiar presentan estimaciones para las 10 ciudades principales (nueve capitales departamentales y El Alto) de manera independiente.

El gasto del hogar se recolectó a partir del método de libreta y un cuestionario de gastos mayores. Las libretas corresponden a gastos diarios del hogar y gastos individuales, llenadas durante siete días de la semana de entrevista. Dichas libretas capturaron gastos en los rubros de alimentación, transporte, productos farmacéuticos, medicinas naturales y otros gastos. El cuestionario de gastos mayores se aplicó a los informantes por recordatorio y se capturaron erogaciones en distintos períodos de referencia, referidos a compras de vestimenta, servicios y mantenimiento de la vivienda, otros gastos en equipamiento del hogar, así como otras transacciones financieras y transferencias que realizan los hogares.

Los resultados de la ECH 2003-2004 evidencian cambios significativos en los patrones de consumo alimentario y gasto familiar con relación a las presentadas a principios de los años noventa. Dichos cambios son atribuidos afactores relacionados con cambiosen precios relativos, mayor diversidad en la oferta de alimentos fuera del hogar y productos envasados, mayor participación de los supermercados y tiendas especializadas poco habituales hace más de una década; así como cambios demográficos significativos que alteraron las pautas de consumo urbano. Para el área rural, es la primera vez que se construye una CBA a partir de la misma fuente que las canastas urbanas ${ }^{3}$.

La valoración de la CBA utilizó los precios al consumidor reportados en la página web del INE 4 . Dichos precios son el promedio de los precios usados para el cálculo del IPC y se encuentran a nivel de variedad y contienen información sobre la especificación de los productos, unidad de medida y equivalencia en cantidad. Los precios anteriores a 2008

individuales. Además se aplicó un cuestionario de gastos mayores y se realizó el seguimiento a la calidad de información general del hogar.

3 Anteriormente la CBA rural correspondía a la Encuesta de Evaluación de Impacto del Fondo de Inversión Social.

4 http://www.ine.gob.bo/ipc/ipcprecios.aspx 
corresponden a los del IPC con base 1991, por lo que existieron productos cuyos precios no fueron recolectados por la diferencia entre las canastas de ambos índices. Los detalles para completar los precios se especifican en Mena (2013) $)^{5}$. Otras fuentes de información incluyen las tablas de conversión de unidades de medida y equivalencias (INE, 1993a). Para la estimación de los valores nutricionales se usaron las tablas bolivianas de composición química de alimentos del Ministerio de Salud y Deporte e INE (2005).

\section{La canasta básica dealimentos}

Las líneas de pobreza son herramientas fundamentales para informar sobre las tendencias de la pobreza. Al definir las canastas básicas alimentarias se considera el detalle de las necesidades medias de energía, pautas de consumo, precios específicos y características socio-demográficas de la población ${ }^{6}$.

La metodología de construcción de las canastas básicas alimentarias toma como referencia la que corresponde a la CEPAL y considera los siguientes aspectos: (a) las necesidades medias de energía de la población, (b) un grupo o población de referencia, (c) la estructura de consumo de la CBA, (d) la evaluación nutricional de la CBA, (e) el costo asociado a la adquisición de la canasta y (f) la actualización del valor de la CBA.

La estructuración de la canasta alimentaria y el establecimiento de los umbrales de pobreza tienen carácter normativo ${ }^{7}$ y prescriptivo para las políticas. Definen quién eso no es pobre; por ello es recomendable tener mayor respaldo en datos observados sobre la base de información referida a las características de la población.

\subsection{Necesidades medias de energía}

La determinación de las necesidades de energía humana toman como referencia los parámetros de necesidades nutricionales elaboradas por FAO-OMS-UNU en 2001. Los requerimientos energéticos se definen a partir de la ingesta de alimentos orientadas al mantenimiento de la salud, de las funciones fisiológicas y el bienestar de las personas;

5 Para mayores referencias véase también el capítulo 7 del "Manual del IPC" OIT (2004) y Feenstra y Diewert (2001).

6 Al contrario, las líneas de pobreza internacionales fijan un estándar, por ejemplo, \$US 1.25 por persona al día, y están afectadas por la paridad de poder de compra. Véase Ravallion, Datt y van de Walle (1991), Ravallion y Chen (1997), Ravallion, Chen y Sangraula (2008).

7 Esto se debe a que las CBAs deben representar el costo de satisfacer estándares nutricionales, lo que es una norma impuesta externamente. 
incluyendo la respuesta a las demandas originadas en actividades sociales. De acuerdo al informe de dichos organismos, el balance de energía se logra cuando la ingesta de energía diaria es igual al gasto de energía más el gasto energético necesario para el crecimiento de los niños, el embarazo y el requerimiento de producir leche durante la lactancia.

Los requerimientos diarios de energía y los niveles de ingesta son recomendaciones promedio sobre un período de tiempo, ello no implica que deban consumirse los montos exactos cada día. La estimación de los requerimientos de energía produce variaciones entre individuos. En consecuencia, existe una distribución de requerimientos dentro de la clase o grupo poblacional.

Con el propósito de facilitar la aplicación de la metodología, FAOy OMS desarrollaron una herramienta de aplicación estandarizada para el cálculo de los requerimientos nutricionales denominada Population Energy Requirement (POPER), que toma en cuenta las proyecciones oficiales de población, las tasas brutas de natalidad y el grado de urbanización proporcionadas por CELADE, asícomo parámetros recomendados sobre el peso corporal de las personas por grupos sexo y edad. Dicha aplicación presenta estimaciones sobre las recomendaciones de energía para países de distinto nivel de desarrollo. La aplicación estándar para Bolivia supone una estructura de población proveniente de las proyecciones demográficas, tasas estimadas de natalidad y supuestos sobre el nivel de actividad física.

Sin embargo, dicha herramienta también permite ajustar parámetros para obtener resultados más precisos. El presente documento introdujo criterios adicionales, entre ellos: (i) utilizó el peso de población por grupos sexo/edad, sugerida por el Ministerio de Salud y Deportes, (ii) se consideraron datos sobre natalidad provenientes de la Encuesta Nacional de Demografía y Salud (ENDSA) de 2003 y 2008, (iii) se consideró que 50\% de la población rural tiene actividad física pesada, mientras que, en el área urbana, dicha proporción es de 25\%, conbase en información de las encuestas de hogares. Con los criterios mencionados, los resultados se encuentran en el cuadro 1. 
Cuadro 1

Requerimientos de energía por persona según área y ciudad

\begin{tabular}{lc}
\hline & Kcal /dia \\
\hline Bolivia & 2123 \\
Urbana & 2094 \\
Rural & 2171 \\
Ciudades $\left({ }^{*}\right)$ & \\
La Paz-EA & 2087 \\
Cochabamba & 2120 \\
Santa Cruz & 2091 \\
Resto urbano & 2099 \\
\hline
\end{tabular}

Fuente: Elaboración de los autores con POPER

La diferencia de requerimientos de energía entre áreas es de casi $80 \mathrm{kcal} /$ día por persona, siendo más alta en el ámbito rural en relación al área urbana. En áreas urbanas se tiene que la dispersión de las kcal es baja, con excepción de la ciudad de Cochabamba. Dada la dispersión relativamente baja de los requerimientos de energía, el presente trabajo adoptó una norma única de 2,123 kcal por persona al día, para cualquier ciudad o área de residencia. Luego del cálculo de los requerimientos de energía, la metodología de la CEPAL propone un procedimiento para determinar la estructura de la CBA a partir de las encuestas de ingresos y gastos.

\subsection{Estimación de nutrientes y estructura del gasto alimentario}

La ECH 2003-2004 capturó el gasto y la cantidad de alimentos consumida en unidades de medida convencionales (arroba, quintal, kilogramo, litro o cc) y en otras no convencionales como: amarro, montón, paquete, bolsa y otros. Por tal motivo, fue necesario adoptar criterios o supuestos sobre la equivalencia y realizar operaciones para la conversión de dichas unidades a gramos a través de tablas elaboradas por entidades nacionales especializadas 8 .

\section{Tratamiento de los datos de la encuesta}

Con el objeto de obtener estimaciones de la estructura de la CBAy evitar sesgos por la presencia de valores extremos en las variables relevantes, se procedió a depurar la encuesta, específicamente en las distribuciones de gasto y cantidades de alimentos declaradas. En

8 Se utilizaron las tablas de conversión de unidades de medida y equivalencias (INE, 1993a). Dicha investigación se basó en la encuesta de seguimiento al consumo alimentario realizada en una región rural de Bolivia en 1991. 
varios casos las libretas registraron gastosy cantidades relacionados con actividades sociales, fiestas u otros eventos. De esta forma se explican algunas cantidades atípicas, de manera que fue necesario depurar los datos de la encuesta a partir de los siguientes criterios: (i) los valores elevados en compras de alimentos por cada artículo diario, especialmente en bebidas alcohólicas y gastos fuera del hogar, se consideraron como otros gastos no alimentarios, (ii) se dejaron de lado las cantidades extremas, debido a que no puede aceptarse ingesta de alimentos por encima de un límite razonable y (iii) se utilizó un método multivariado para la detección de outliers?

Una vez determinadas las cantidades físicas de los alimentos expresadas en gramos o cc., se aplicó el factor de corrección con el propósito de establecer la cantidad comestible o peso neto de dichos alimentos. El factor de corrección es un coeficiente que tiene la función de evitar la sobreestimación del valor nutritivo de los alimentos y mide la pérdida de aquella parte de los alimentos que deriva de las prácticas comunes de preparación de alimentos en Bolivia, las pérdidas que provienen de los procedimientos manuales y la utilización de la ayuda de instrumentos cortantes. El desecho comprende la porción no comestible (cáscaras, semillas, residuos de tierra y otros) que se encuentran implícitos en el peso de venta.

Para los alimentos consumidos fuera del hogar se adoptaron criterios complementarios que desarrolló el INE con información proveniente de una encuesta simplificada de consumo, que serealizó en restaurantes y puestos deventa de comidas en La Paz, Cochabamba y Santa Cruz.

Luego de calcular el peso neto de los alimentos, se determinó el contenido de los nutrientes correspondientes a las cantidades de alimentos a partir de la utilización de las tablas de composición química de alimentos elaboradas por entidades nacionales ${ }^{10}$.

Se creó una nueva base de datos con las estimaciones de las cantidades per cápita por persona al día y la correspondiente equivalencia en términos de los nutrientes por cada producto. La lista completa de alimentos se clasificó según la siguiente agrupación, con

9 Para la detección de valores extremos o outliers, CEPAL utilizó un método multivariado basado en la distancia cuadrática de Mahalanobis (D2). Dicho método consiste en la estimación de una variable de interés y la predicción de los errores al cuadrado. El valor-p asociado al error de estimación es un indicador de la confiabilidad de la distancia entre el valor observado y la predicción, de manera que genera una estimación de los valores más alejados de la predicción.

10 "Tablas bolivianas de composición química de alimentos crudos", del Ministerio de Salud y Deportes e INE. Dicho trabajo tiene como base la investigación de la encuesta de consumo de alimentos de 1991. 
el propósito de diferenciar el origen y la función de cada producto en la alimentación humana ${ }^{11}$ :

Subgrupo 1. Pan y cereales

Subgrupo 2. Carnes, menudencias y embutidos

Subgrupo 3. Pescados

Subgrupo 4. Aceites y grasas

Subgrupo 5. Leche, derivados y huevos

Subgrupo 6. Verduras frescas y en conserva

Subgrupo 7. Tubérculos, raíces y derivados

Subgrupo 8. Leguminosas

Subgrupo 9. Frutas frescas, secas y en conserva

Subgrupo 10.Azúcar, mermeladas y miel

Subgrupo 11. Café, té, cacao e infusiones

Subgrupo 12. Productos alimenticios diversos

Subgrupo 13. Aguas minerales, refrescos, jugos de frutas

Subgrupo 14. Bebidas destiladas alcohólicas

Subgrupo 15. Alimentos fuera del hogar

Subgrupo 16. Bebidas consumidas fuera del hogar

\subsection{Definición de la población de referencia}

De acuerdo con CEPAL, la CBA corresponde a las pautas de consumo de un grupo de referencia de la población, en particular aquellos hogares que razonablemente alcanzan una adecuación energética por encima de los niveles recomendados. La definición de un grupo de población de referencia tiene la función de proveer una estructura inicial del consumo alimentario de la población. Por varios años CEPAL mantuvo el supuesto de que la CBA debía reflejar las pautas de consumo de un grupo de la población que no tuviese restricciones significativas de recursos para la adquisición de alimentos alos precios vigentes. El tamaño del estrato de referencia garantiza cierta representatividad y, al mismo tiempo,

11 La clasificación tiene correspondencia con la agrupación propuesta por Naciones Unidas para el Sistema de Cuentas Nacionales (SCN). 
tiene la función de excluir a los hogares con ingresos extremos (altos y bajos), de manera que incorpora a los hogares ubicados en una posición intermedia en la distribución del ingreso.

El presente trabajo mantiene los criterios de CEPAL para la obtención del grupo de referencia específico para cada dominio de estudio. Definimos un índice de adecuación energética para los $k$ dominios como:

$$
\operatorname{Iae}_{i k}=\frac{\overline{K_{c a l}}}{2,123} * 100 \quad i \in[1,81] ; \quad k \in[1,5]
$$

donde $\overline{K_{c a l}}$ es el promedio de kilo calorías (energía) consumida por el quintil móvil $i$ del gasto en consumo per capita ${ }^{12}$ del dominio $k$.

Entonces, la población de referencia se compone del primer quintil móvil que en promedio supera el $100 \%$ de este índice. Visto de otra forma, la población de referencia estará compuesta por el primer quintil móvil de gasto en consumo per cápita del hogar que alcanza una adecuación energética promedio igual a las recomendaciones promedio. Para un estudio del impacto del uso de criterios alternativos en la elección de la población de referencia véase Feres (s/f).

Figura 1: Índice de adecuación energética

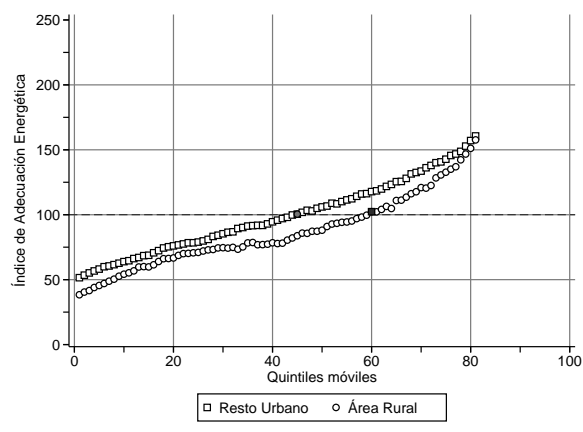

(a) Resto urbano y área rural

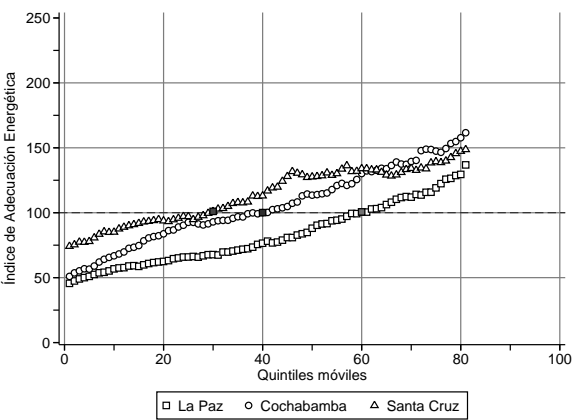

(b) Ciudades capitales

Fuente: Elaboración propia con base en la ECH 2003-2004 (INE)

12 El quintil móvil se construye dividiendo a la población en cada dominio en 100 percentiles, de acuerdo al gasto del hogar per cápita; luego se realizan promedios móviles de cada grupo de 20 percentiles. 
Los paneles de la figura 1 muestran la distribución de la adecuación energética en cada uno de los dominios de estudio para la obtención del estrato de población de referencia. La definición específica de los estratos de población se presenta en el cuadro 2.

\section{Cuadro 2}

\section{Estrato de referencia según gasto per cápita}

\begin{tabular}{lccc}
\hline & $\begin{array}{l}\text { Percentiles de hogares según } \\
\text { gasto per capita }\end{array}$ & $\begin{array}{l}\text { Índice de Adecuación Calóri- } \\
\text { ca Promedio (\%) }\end{array}$ & Valor por mil calorías (Bs.) \\
\hline Resto urbano & $45-64$ & 100.46 & 2.44 \\
La Paz - El Alto & $60-79$ & 100.63 & 2.92 \\
Cochabamba & $40-59$ & 100.08 & 2.65 \\
Santa Cruz & $30-49$ & 101.06 & 2.25 \\
Área rural & $60-79$ & 102.34 & 2.02 \\
\hline
\end{tabular}

Fuente: Elaboración propia a partir de datos de la ECH 2003 - 2004 (INE)

Como se mencionó anteriormente, el grupo de referencia es el primer quintil móvil que alcanza una adecuación calórica promedio superior al 100\%, de manera que los hogares incorporados en el grupo de referencia presentan una suficiencia de energía.

El procedimiento de selección del estrato de referencia considera el grado de eficiencia con que aquellos hogares alcanzan la adecuación energética: los hogares son muy eficientes si el estrato de población se encuentra en la parte de bajos ingresos, y son menos eficientes cuando la población de referencia sehalla en la parte de ingresos más altos. Ello se observa en la distribución del costo por 1000 calorías en la figura 2, de manera que los hogares con menores ingresos tienden a cubrir las necesidades energéticas con menor costo, y éste se eleva a medida que se incrementan los ingresos.

Figura 2: Costo por mil calorías (en Bs.)

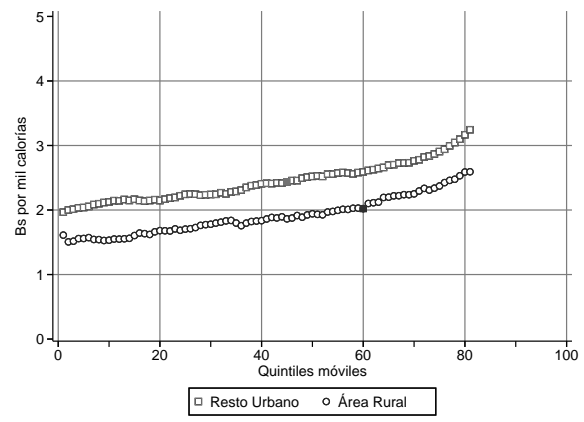

(a) Resto urbano y área rural

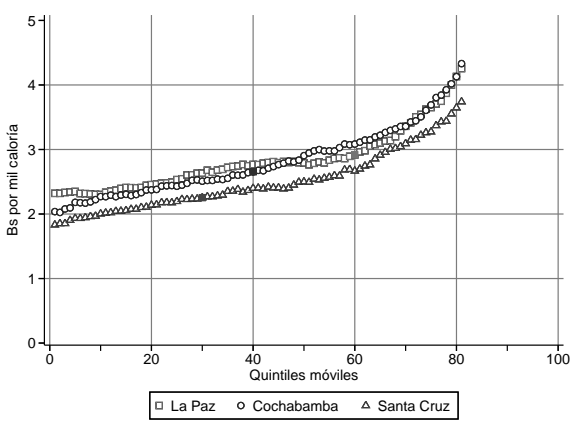

(b) Ciudades capitales

Fuente: Elaboración propia con base en la ECH 2003-2004 (INE) 
La distribución del costo por caloría no muestra diferencias significativas entre áreas ni entre ciudades. En el área rural, la adquisición de calorías tiende a ser inferior respecto a las ciudades consideradas en el resto urbano, lo que probablemente se atribuya al componente de autoconsumo y menores márgenes de comercialización de alimentos agropecuarios en los lugares de compra.

\section{Selección de artículos de la CBA}

Una vez definida la población de referencia en cada dominio de estudio, en este caso ordenado por el gasto del hogar per cápita, se procede a determinar una lista de los alimentos representativos y relacionados con los patrones de consumo de la población de referencia. La administración de la CBA debe manejar los artículos básicos que consume la población de referencia. La selección de los productos de la CBA se realizó en función a los siguientes criterios:

- Tener una frecuencia de compra de al menos $25 \%$ de los hogares de referencia.

- Tener una participación de al menos 2\% del gasto alimentario total.

- Contribuir por lo menos con $1 \%$ a la ingesta de calorías totales.

- Tener una participación de al menos 5\% en el gasto del subgrupo.

- Estar incluidos en la canasta del IPC base 2007.

Hubo varios artículos en cada subgrupo que no cumplieron los criterios de selección, los cuales se consolidaron en un genérico denominado "otros", de manera que el gasto y el aporte de calorías fueron distribuidos proporcionalmenteentre los que síse seleccionaron. De esta forma, la estructura de la CBA mantiene las pautas de consumo de los hogares del grupo de referencia, tanto en la distribución del gasto como en las cantidades físicas, tal como recomiendan los procedimientos usuales de CEPAL para especificar las cantidades físicas de las canastas alimentarias.

Las diferencias entre la estructura del promedio del consumo de la población y del estrato de referencia señalan las discrepancias entre las pautas de consumo de la población en su conjunto y la población incluida en el estrato de referencia que efectivamente presentan una adecuación energética cercana a 100\%. En este sentido, la CBA se acerca más 
hacia un criterio normativo y verifica con mayor precisión la satisfacción de necesidades alimentarias.

Con la selección de alimentos, la estructura de consumo se redujo desde más de 200 artículos capturados por la ECH 2003-2004, hasta aproximadamente 60 artículos, manteniendo la estructura y los hábitos de consumo de la población de referencia. La CBA se ajustó hasta que las cantidades y el aporte nutricional del conjunto de ellos igualen a las recomendaciones de energía.

\subsection{Evaluación de la calidad nutricional de las CBA}

La CBA no sólo satisface los requerimientos de calorías y proteínas, sino que también requiere que tenga una composición capaz de cumplir con otros estándares dietéticos.

\section{Distribución del aporte calórico}

El aporte calórico o molécula calórica es la distribución porcentual de energía proporcionada por los tres macronutrientes: proteínas, hidratos de carbono y grasas, que se muestran en el cuadro 3. Se considera aceptable una dieta que, en promedio, tenga al menos $10 \%$ de calorías proteicas. El rango de calorías grasas es entre 15 y $20 \%$. Asimismo, la CBA establece que los hidratos de carbono, aproximados por el contenido de cereales y leguminosas, significan entre 30\% y 50\% de las calorías totales de la dieta.

\section{Cuadro 3 \\ Evaluación nutricional de la canasta básica de alimentos}

\begin{tabular}{lccc}
\hline & \multicolumn{3}{c}{ Molécula calórica } \\
& Proteínas & Grasas & Carbohidratos \\
\hline Resto urbano & 11.98 & 17.43 & 44.36 \\
La Paz - El Alto & 12.42 & 17.56 & 37.88 \\
Cochabamba & 13.81 & 18.96 & 36.51 \\
Santa Cruz & 12.59 & 16.35 & 48.97 \\
Área rural & 11.83 & 16.53 & 48.01 \\
\hline
\end{tabular}

Fuente: Elaboración propia a partir de datos de la ECH 2003 - 2004 (INE)

\section{Origen de las proteínas}

El origen de las proteínas se muestra en el cuadro 4.En cuanto a la calidad de las proteínas, se estimó adecuado que entre 30y 40\% de las mismas fuera de origen animal (CEPAL, 1991). 
Las CBA de los distintos dominios de estudio mantienen un rango razonable, con un sesgo hacia el límite inferior en cuanto al origen de las proteínas animales.

\section{Cuadro 4 \\ Orígen de las proteínas contenidas en la CBA}

\begin{tabular}{lcc}
\hline & \multicolumn{2}{c}{ Origen de las Proteínas (\%) } \\
& Animal & Vegetal \\
\hline Resto urbano & 29.42 & 70.58 \\
La Paz - El Alto & 30.01 & 69.99 \\
Cochabamba & 32.53 & 67.47 \\
Santa Cruz & 26.44 & 73.56 \\
Área rural & 25.89 & 74.11 \\
\hline
\end{tabular}

Fuente: Elaboración propia a partir de datos de la ECH 2003-2004 (INE)

\subsection{Estructura de las canastas básicas de alimentos}

En los cuadros 5 y 6 se documentan las cantidades consumidas según subgrupos de alimentos. La estructura de la CBA de la ciudad de La Paz muestra una mayor ponderación de alimentos del grupo de tubérculos, raíces y derivados, y un bajo consumo de leches, derivados y huevos. A pesar de ello, muestra cambios respecto a la estructura de la dieta estimada en años anteriores ${ }^{13}$.

En la ciudad de Cochabamba, el porcentaje de la CBAdedicado a verduras frescas es de alrededor del13\%, el cual es superior al resto de los dominios. Asimismo, la ponderación de las frutas frescas en la CBA es superior en un 1\% en relación a La Paz y 5\% en relación a Santa Cruz. Probablemente esto se deba a que en Cochabamba los precios de los productos contenidos en los subgrupos son menores debido a factores climáticos, y por lo tanto su consumo es mayor.

Por otra parte, se observa que la población de referencia en la ciudad de Santa Cruz de la Sierra concentra gran parte de la dieta en tres tipos de alimentos: pan y cereales, carnes y el grupo de alimentos y bebidas consumidas fuera del hogar; la representatividad de dichos grupos en el consumo alimentario es superior al resto de las ciudades analizadas; en cambio, el consumo de verduras y tubérculos es comparativamente menor a la del resto. La explicación de esta estructura obedece a las diferencias en precios relativos, toda vez

13 En 1990, los datos para la ciudad de El Alto con la EPF (INE, 1994) mostraron con la muestra total que cerca del $24 \%$ de las cantidades correspondían a pan y cereales. Esta proporción disminuyó en 2003-2004, al contrario, sólo $4 \%$ correspondía a carnes. Dichos cambios manifiestan una diversificación de la dieta. 
que verduras y carnes tienen menor costo relativo; además, un mayor consumo de carnes implica que la población puede alcanzar los requerimientos calóricos y proteínicos con menor cantidad de alimentos.

\section{Cuadro 5}

Cantidades al día de la CBA por ciudad, según grupos de alimentos (gramos o cc)

\begin{tabular}{|c|c|c|c|c|c|c|}
\hline \multirow[t]{2}{*}{ Grupo de Alimentos } & \multicolumn{2}{|c|}{ La Paz } & \multicolumn{2}{|c|}{ Cochabamba } & \multicolumn{2}{|c|}{ Santa Cruz } \\
\hline & Cantidad pc & $\%$ & Cantidad pc & $\%$ & Cantidad pc & $\%$ \\
\hline Alimentos y Bebidas & 1118.68 & 100.00 & 1238.27 & 100.00 & 981.95 & 100.00 \\
\hline Pan y Cereales & 241.06 & 21.55 & 236.15 & 19.07 & 328.70 & 33.47 \\
\hline Carnes, menudencias y embutidos & 72.36 & 6.47 & 72.77 & 5.88 & 69.40 & 7.07 \\
\hline Pescado & 5.89 & 0.53 & 0.91 & 0.07 & 1.07 & 0.11 \\
\hline Aceites y grasas & 16.96 & 1.52 & 17.10 & 1.38 & 12.88 & 1.31 \\
\hline Leche, derivados y huevos & 38.76 & 3.46 & 74.86 & 6.05 & 59.34 & 6.04 \\
\hline Verduras frescas y en conserva & 117.09 & 10.47 & 167.82 & 13.55 & 79.74 & 8.12 \\
\hline Tubérculos, raíces y derivados & 127.12 & 11.36 & 138.35 & 11.17 & 60.18 & 6.13 \\
\hline Leguminosas & 10.43 & 0.93 & 14.09 & 1.14 & 3.34 & 0.34 \\
\hline Frutas frescas, secas y en conserva & 93.57 & 8.36 & 117.21 & 9.47 & 42.07 & 4.28 \\
\hline Azúcar, mermeladas y miel & 42.45 & 3.79 & 34.15 & 2.76 & 38.79 & 3.95 \\
\hline Café,Te, Cacao e infusiones & 3.93 & 0.35 & 1.90 & 0.15 & 3.30 & 0.34 \\
\hline Productos alimenticios diversos & 7.96 & 0.71 & 6.79 & 0.55 & 7.81 & 0.80 \\
\hline Agua mineral, refrescos, jugos de frutas & 98.23 & 8.78 & 95.00 & 7.67 & 24.45 & 2.49 \\
\hline Bebidas destiladas alcohólicas & 3.95 & 0.35 & 4.39 & 0.35 & 0.43 & 0.04 \\
\hline Alimentos consumidos fuera del hogar & 188.68 & 16.87 & 162.59 & 13.13 & 205.72 & 20.95 \\
\hline Bebidas consumidas fuera del Hogar & 50.21 & 4.49 & 94.20 & 7.61 & 44.71 & 4.55 \\
\hline
\end{tabular}

Fuente: Elaboración propia a partir de datos de la ECH 2003-2004 (INE)

La estructura de la CBA en el resto urbano y el área rural presenta aspectos relevantes; en este último el consumo aparente de carne es menor y se reemplaza con el consumo proporcionalmente más alto en el grupo de tubérculos y raíces. El gasto de alimentos y bebidas fuera del hogar es bastante más bajo en el área rural, en correspondencia con la menor oferta de esta modalidad en áreas dispersas. 
Cuadro 6

Cantidades al día de la CBA por área, según grupos de alimentos (gramos o cc)

\begin{tabular}{|l|rrrr|}
\hline Grupo de Alimentos & \multicolumn{2}{|c}{ Resto Urbano } & \multicolumn{3}{c|}{ Área Rural } \\
& Cantidad pc & $\%$ & Cantidad pc & $\%$ \\
\hline Alimentos y Bebidas & 1069.87 & 100.00 & 959.70 & 100.00 \\
Pan y Cereales & 286.46 & 26.78 & 295.67 & 30.81 \\
Carnes, menudencias y embutidos & 62.39 & 5.83 & 47.13 & 4.91 \\
Pescado & 2.45 & 0.23 & 6.08 & 0.63 \\
Aceites y grasas & 19.51 & 1.82 & 22.87 & 2.38 \\
Leche, derivados y huevos & 30.38 & 2.84 & 25.88 & 2.70 \\
Verduras frescas y en conserva & 144.26 & 13.48 & 108.27 & 11.28 \\
Tubérculos, raíces y derivados & 153.74 & 14.37 & 167.79 & 17.48 \\
Leguminosas & 9.83 & 0.92 & 10.07 & 1.05 \\
Frutas frescas, secas y en conserva & 68.45 & 6.40 & 57.18 & 5.96 \\
Azúcar, mermeladas y miel & 44.82 & 4.19 & 51.71 & 5.39 \\
Café,Te, Cacao e infusiones & 3.65 & 0.34 & 4.84 & 0.50 \\
Productos alimenticios diversos & 12.81 & 1.20 & 16.95 & 1.77 \\
Agua mineral, refrescos, jugos de frutas & 52.52 & 4.91 & 29.17 & 3.04 \\
Bebidas destiladas alcohólicas & 3.42 & 0.32 & 7.17 & 0.75 \\
Alimentos consumidos fuera del hogar & 114.54 & 10.71 & 63.09 & 6.57 \\
Bebidas consumidas fuera del Hogar & 60.62 & 5.67 & 45.83 & 4.78 \\
\hline
\end{tabular}

Fuente: Elaboración propia a partir de datos de la ECH 2003 - 2004 (INE)

Las canastas cubren los requerimientos de energía promedio de la población en los distintos ámbitos geográficos evaluados. La ingesta de calorías está distribuida entre los grupos de alimentos, y reflejan la distribución del consumo alimentario, las preferencias por ciertos grupos alimentarias y las condiciones de la oferta. La estructura de la CBA en los distintos dominios de estudio reflejan las diferencias en las pautas de consumo alimentario en las regiones evaluadas. Sin embargo, dichas CBA también son normativas, por cuanto se ajustaron para cubrir los requerimientos nutricionales con base en parámetros deseables desde el punto de vista de la ingesta.

En el área metropolitana de La Paz (ciudades de La Pazy El Alto), más del 75\% de las calorías de la estructura de la CBA provienen del consumo de alimentos dentro del hogar. Destacan principalmente las calorías originadas en el consumo de pan y cereales, que contribuyen con 37\%. Es destacable la participación de panes y cereales en la ciudad de Santa Cruz de la Sierra, que aporta casi $48 \%$ por encima del resto de las ciudades analizadas. 


\section{Cuadro 7}

Calorías al día de la CBA por ciudad según grupos de alimentos

\begin{tabular}{|l|rrrrrr|}
\hline Grupo de Alimentos & \multicolumn{2}{|c}{ La Paz } & \multicolumn{2}{c}{ Cochabamba } & \multicolumn{2}{c|}{ Santa Cruz } \\
& Kcal pc & $\%$ & Kcal pc & $\%$ & Kcal pc & \% \\
\hline Alimentos y Bebidas & 2123.00 & 100.00 & 2123.00 & 100.00 & 2123.00 & 100.00 \\
Pan y Cereales & 791.51 & 37.28 & 760.05 & 35.80 & 1033.36 & 48.67 \\
Carnes, menudencias y embutidos & 118.03 & 5.56 & 148.67 & 7.00 & 113.03 & 5.32 \\
Pescado & 8.13 & 0.38 & 1.59 & 0.08 & 2.30 & 0.11 \\
Aceites y grasas & 148.55 & 7.00 & 151.58 & 7.14 & 113.62 & 5.35 \\
Leche, derivados y huevos & 52.70 & 2.48 & 78.48 & 3.70 & 54.38 & 2.56 \\
Verduras frescas y en conserva & 43.07 & 2.03 & 62.14 & 2.93 & 30.53 & 1.44 \\
Tubérculos, raíces y derivados & 169.10 & 7.97 & 172.81 & 8.14 & 76.19 & 3.59 \\
Leguminosas & 12.69 & 0.60 & 15.02 & 0.71 & 6.34 & 0.30 \\
Frutas frescas, secas y en conserva & 74.70 & 3.52 & 93.37 & 4.40 & 37.85 & 1.78 \\
Azúcar, mermeladas y miel & 161.53 & 7.61 & 122.84 & 5.79 & 153.01 & 7.21 \\
Café,Te, Cacao e infusiones & 7.09 & 0.33 & 4.97 & 0.23 & 4.15 & 0.20 \\
Productos alimenticios diversos & 6.97 & 0.33 & 6.94 & 0.33 & 6.30 & 0.30 \\
Agua mineral, refrescos, jugos de frutas & 43.40 & 2.04 & 39.91 & 1.88 & 13.24 & 0.62 \\
Bebidas destiladas alcohólicas & 3.17 & 0.15 & 2.86 & 0.13 & 0.30 & 0.01 \\
Alimentos consumidos fuera del hogar & 423.14 & 19.93 & 346.49 & 16.32 & 418.80 & 19.73 \\
Bebidas consumidas fuera del Hogar & 59.20 & 2.79 & 115.28 & 5.43 & 59.60 & 2.81 \\
\hline
\end{tabular}

Fuente: Elaboración propia a partir de datos de la ECH 2003-2004 (INE)

Laestructura delaCBAen elresto urbano yruraltambiénpresenta una alta concentración del aporte de pan y cereales, y especialmente en área rural destaca el aporte calórico de tubérculos, raíces y derivados, que podría estar compensando por una mayor ingesta de calorías provenientes de aceites y grasas. A su vez, este último resultado se explica por la menor incorporación de energía atribuida al consumo fuera del hogar, que normalmente tiene un alto contenido de grasas y calorías.

La estructura de las CBA manifiesta una elevada variabilidad en la distribución de cantidades y calorías entre los grupos de alimentos identificados. A pesar de ello, el presente trabajo agregó las pautas de consumo del resto urbano como uno solo, así como también del área rural en su conjunto, entendiendo que aún no se dispone de la información sobre los precios de los alimentos y especialmente por la mayor ponderación poblacional de las cuatro ciudades evaluadas. 


\section{Cuadro 8 \\ Calorías al día de la canasta básica de alimentos por área según grupos de alimentos}

\begin{tabular}{|l|rrrr|}
\hline Grupo de Alimentos & \multicolumn{2}{|c}{ Resto Urbano } & \multicolumn{2}{c|}{ Área Rural } \\
& Kcal pc & $\%$ & Kcal pc & $\%$ \\
\hline Alimentos y Bebidas & 2123.00 & 100.00 & 2123.00 & 100.00 \\
Pan y Cereales & 927.38 & 43.68 & 999.06 & 47.06 \\
Carnes, menudencias y embutidos & 117.79 & 5.55 & 91.41 & 4.31 \\
Pescado & 4.12 & 0.19 & 10.14 & 0.48 \\
Aceites y grasas & 172.45 & 8.12 & 204.91 & 9.65 \\
Leche, derivados y huevos & 44.76 & 2.11 & 39.84 & 1.88 \\
Verduras frescas y en conserva & 57.05 & 2.69 & 61.99 & 2.92 \\
Tubérculos, raíces y derivados & 194.94 & 9.18 & 238.99 & 11.26 \\
Leguminosas & 14.37 & 0.68 & 20.13 & 0.95 \\
Frutas frescas, secas y en conserva & 58.53 & 2.76 & 53.56 & 2.52 \\
Azúcar, mermeladas y miel & 170.01 & 8.01 & 201.90 & 9.51 \\
Café,Te, Cacao e infusiones & 6.69 & 0.32 & 10.27 & 0.48 \\
Productos alimenticios diversos & 6.46 & 0.30 & 2.97 & 0.14 \\
Agua mineral, refrescos, jugos de frutas & 25.51 & 1.20 & 14.98 & 0.71 \\
Bebidas destiladas alcohólicas & 3.96 & 0.19 & 6.29 & 0.30 \\
Alimentos consumidos fuera del hogar & 248.26 & 11.69 & 127.69 & 6.01 \\
Bebidas consumidas fuera del Hogar & 70.72 & 3.33 & 38.88 & 1.83 \\
\hline
\end{tabular}

Fuente: Elaboración propia a partir de datos de la ECH 2003-2004 (INE)

\section{Valoración de la CBA}

Una vez que se cuenta con la estructura de la canasta de alimentos, se procede a su valoración y actualización durante el periodo de estudio. Para la valoración de la CBA se usaron los precios promedio para el cálculo del IPC, con base 2007. Los precios del nuevo IPC son recolectados para todas las ciudades capitales de departamento más la ciudad de El Alto. Esto permite la valoración de las CBA a partir de las cantidades estimadas y los precios del IPC.

Los precios tenían la especificación del producto, lo que permitió en muchos casos contar con la cantidad y transformarla a gramos o cc. También existieron productos que no reportaban una unidad de medida del sistema internacional, y en ese caso se procedió a estimar la cantidad a través de las tablas de conversión. Sin embargo, la estimación del valor de la CBA en el área rural tiene una severa dificultad, ya que el IPC no recolecta precios para el área rural.

Para estimar las diferencias entre el costo de una CBA entre el área urbana y el área rural se procedió a estimar las diferencias de costo de un conjunto homogéneo de productos valorados en el área rural, respecto al valor de los mismos artículos en el área urbana. Dicho 
coeficiente corresponde al grupo de referencia de cada dominio, y se mantiene constante durante el período de análisis. Los resultados de la valoración y la actualización de las canastas básicas de alimentos se visualizan en la figura 3. En el cuadro 9 se documenta el valor de la línea de pobreza para el mes de diciembre de cada año.

Figura 3: Evolución del costo de la CBA según dominio (en Bs.)

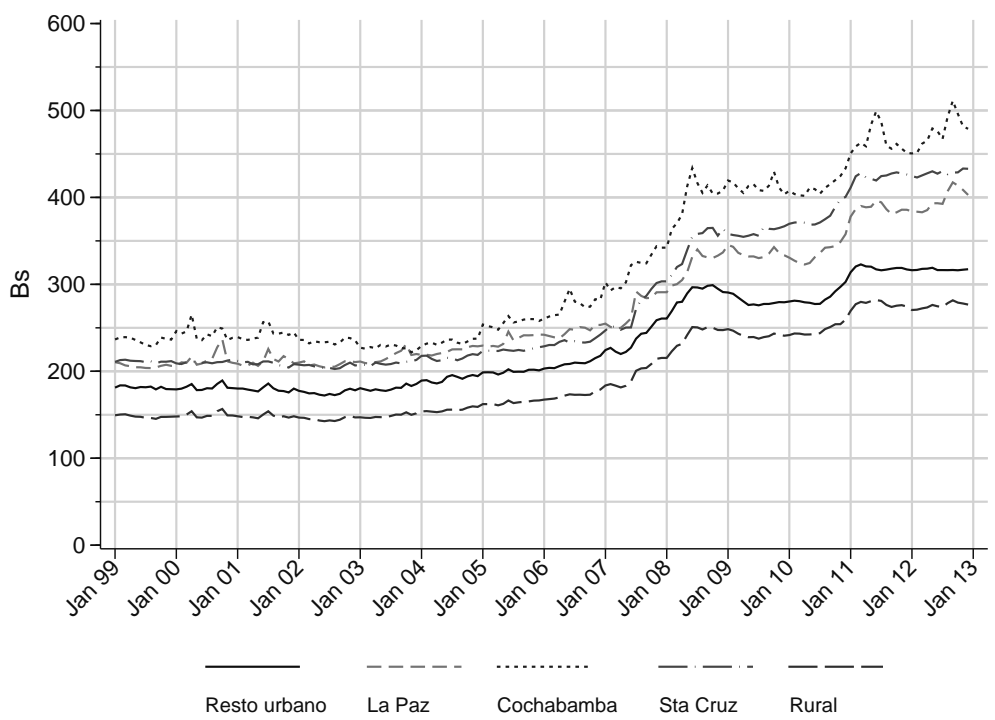

Fuente: Elaboración propia con base en la ECH 2003-2004 y precio del IPC (INE)

\section{Cuadro 9}

Línea de indigencia a diciembre, según dominio (en Bs. por persona al mes)

\begin{tabular}{|l|rrrrr|}
\hline Fecha & Resto Urbano & La Paz & Cochabamba & Santa Cruz & Rural \\
\hline Dic 99 & 179.38 & 206.26 & 236.51 & 211.70 & 147.82 \\
Dic 00 & 180.63 & 209.78 & 238.49 & 213.46 & 149.17 \\
Dic 01 & 180.10 & 209.15 & 244.59 & 208.28 & 148.29 \\
Dic 02 & 178.16 & 210.63 & 235.56 & 206.71 & 146.99 \\
Dic 03 & 185.47 & 219.89 & 224.23 & 213.12 & 151.71 \\
Dic 04 & 194.47 & 228.73 & 237.61 & 218.87 & 159.06 \\
Dic 05 & 201.06 & 242.28 & 257.90 & 227.66 & 166.49 \\
Dic 06 & 217.82 & 253.36 & 282.46 & 242.22 & 178.99 \\
Dic 07 & 260.70 & 290.97 & 342.22 & 303.46 & 215.39 \\
Dic 08 & 291.16 & 336.70 & 407.76 & 363.83 & 247.39 \\
Dic 09 & 279.38 & 333.47 & 403.56 & 366.76 & 240.94 \\
Dic 10 & 302.78 & 357.69 & 433.30 & 400.57 & 259.44 \\
Dic 11 & 317.15 & 385.81 & 451.28 & 426.27 & 274.46 \\
Dic 12 & 317.42 & 402.85 & 478.93 & 432.93 & 276.77 \\
\hline
\end{tabular}

Fuente: Elaboración propia con base en la ECH 2003 - 2004 y precio del IPC (INE) 
$\mathrm{Al}$ analizar las tendencias, se observa en primera instancia que el valor de la CBA se ha incrementado en todos los dominios. Un caso que llama la atención son las divergencias en el costo de la canasta entre La Paz y Santa Cruz. Aproximadamente hasta mediados de 2008 la evolución de las canastas había sido similar; después de 2008 las diferencias se hicieron más amplias llegando a una diferencia máxima de Bs. 48 en abril y noviembre de 2010.

También llama la atención el nivel del valor de la canasta de alimentos en el área rural, la cual se encuentra por debajo del resto de los dominios. Posiblemente esto se deba a que en el área rural, al ser un área mayoritariamente dedicada a la actividad agrícola, el valor de los alimentos sea menor en relación al área urbana. La ciudad de Cochabamba tiene el costo de CBA más alto, debido, entre otras cosas, a que la cantidad de la canasta es mayor en relación al resto de los dominios. Finalmente, se tiene que en el periodo 2007-2008 el precio de las canastas en todos los dominios seincrementó notoriamente, debido aque fue un periodo de inflación de alimentos en el país.

\subsection{Comparando las líneas de pobreza extrema}

Si bien estos resultados son interesantes por sí mismos, es preciso compararlos con otras líneas de pobreza extrema. Esto nos dará una primera imagen de las implicaciones de usar diferentes líneas de pobreza. En la figura 4 segraficaron los valores de la línea de pobreza extrema estimada para los meses en los que se llevó a cabo el operativo de la encuesta de hogares en 1999, 2005 y el periodo posterior a 2009. Adicionalmente se reportan entre paréntesis las diferencias entre las líneas estimadas y las oficiales.

Se puede apreciar que las mayores diferencias entre las líneas se dan en Cochabamba, donde la diferencia llega a ser de Bs. 77 en 2009, por ejemplo. Por otra parte, las diferencias más pequeñas se dan en el área rural, donde, por ejemplo, en 2009 la diferencia llega a ser tan sólo de 40 centavos de Boliviano. Estos resultados implican que las medidas de pobreza a nivel urbano serán mayores, lo que también afectará la tasa de evolución de la pobreza extrema en el área urbana. Por otra parte, la semejanza en la evolución de la línea de pobreza extrema en el área rural implicará que probablemente lo niveles de pobreza no sufrirán mayores cambios. Este resultado es sumamente importante, dado que trabajos recientes (Hernani y Eid, 2013) muestran que es en el área rural donde los ingresos reales crecieron a una mayor velocidad en relación al área urbana. 
Figura 4: Diferencias entre las líneas de indigencia estimadas y las oficiales

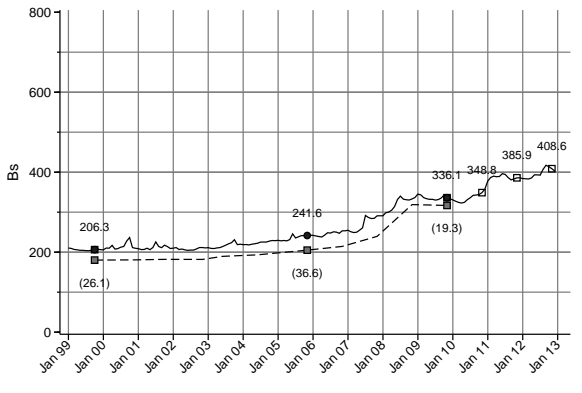

(a) La Paz

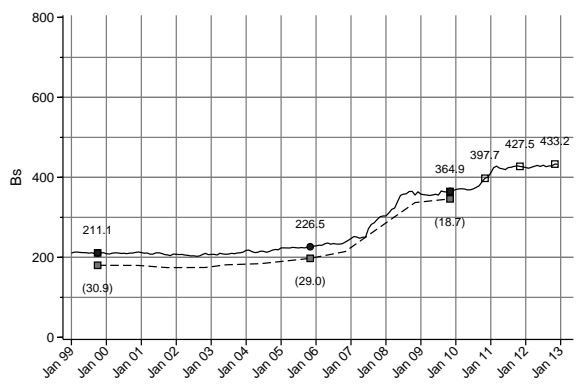

(c) Santa Cruz

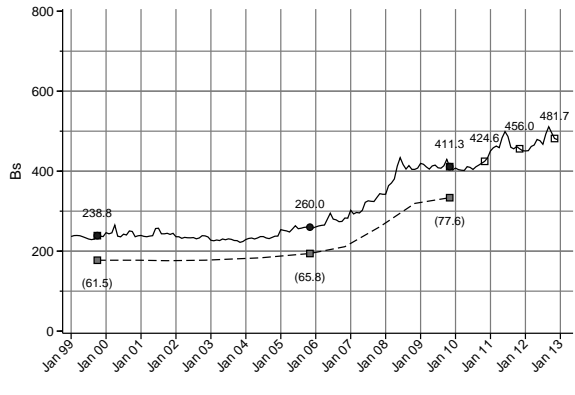

(b) Cochabamba

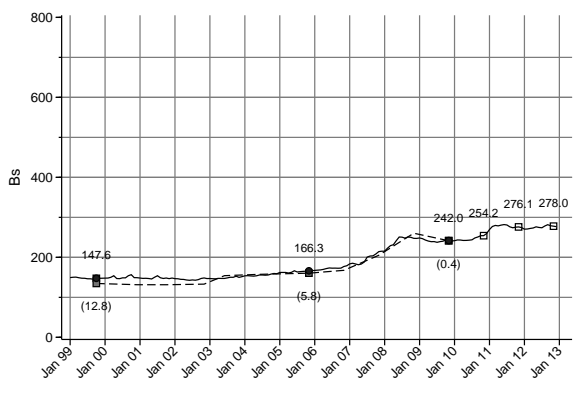

(d) Área rural

Fuente: Elaboración propia con base en la ECH 2003 - 2004 y datos de UDAPE

Frecuentemente las mediciones de pobreza usan como referencia la línea de pobreza extrema internacional (\$US 1.25 en PPP) para hacer comparaciones de pobreza entre países. Un detalle a considerar es que existen dos tasas diferentes de tipo de cambio a PPP. Por un lado se tiene la tasa de cambio calculada por el Programa Internacional de Comparaciones (ICP, por sus siglas en inglés), cuya metodología se encuentra descrita en Banco Mundial (2008). Por el otro, uno puede usar el tipo de cambio a PPP calculado en el Penn World Table (PWT), cuya metodología se encuentra en Feenstra y Diewert (2013).

Las metodologías del ICP y del PWT muestran resultados distintos en el tipo de cambio de Bolivianos a PPP, lo que explica las diferencias en la figura 5. Estas diferencias pueden conducir a estimaciones de pobreza distintas, ya que hasta 2005 el tipo de cambio de Bs.a PPP calculado por el ICP esmayor en relación al de PWT, y por ende las medidas de 
pobreza calculadas con el PPP del ICP debería ser mayores. Después de 2005 se produce una inversión, ya que el tipo de cambio de Bs. a PPP de PWT es mayor al del ICP.Como muestra el cuadro 10, la brecha entre ambas estimaciones de PPP llega a una diferencia máxima de 61 décimas en 2011.

Figura 5: Evolución del tipo de cambio a PPP

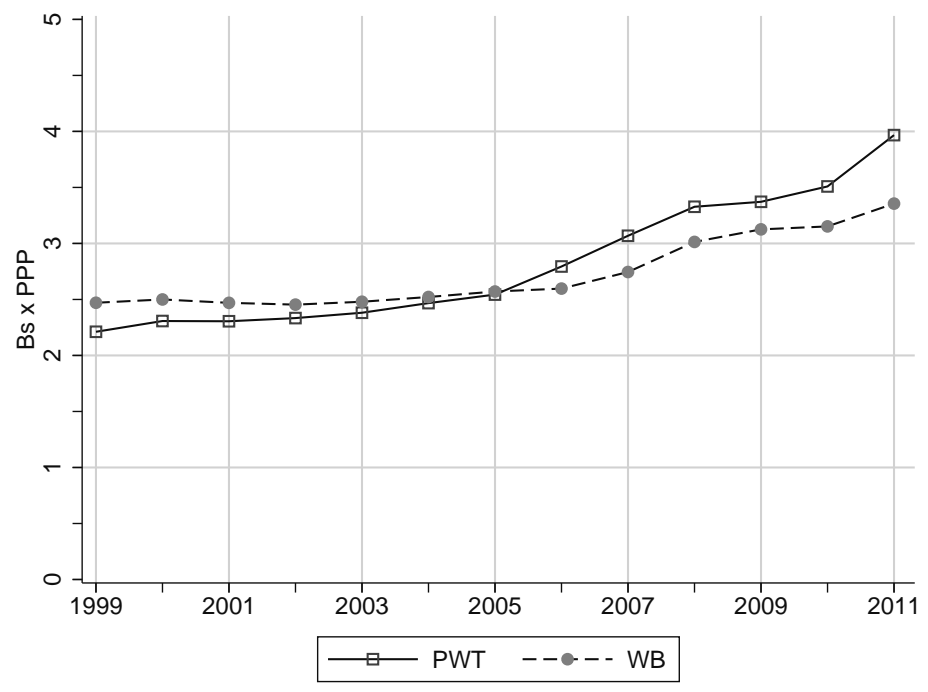

Fuente: Elaboración propia en base a datos de PWT v8.0 y Banco Mundial

Cuadro 10

Tipo de cambio de Bs. $x$ PPP, según fuente

\begin{tabular}{|c|c|c|c|c|c|c|c|c|c|c|c|c|c|}
\hline & 1999 & 2000 & 2001 & 2002 & 2003 & 2004 & 2005 & 2006 & 2007 & 2008 & 2009 & 2010 & 2011 \\
\hline PWT v8.0 & 2.21 & 2.31 & 2.31 & 2.33 & 2.38 & 2.47 & 2.54 & 2.79 & 3.07 & 3.33 & 3.37 & 3.51 & 3.97 \\
\hline Banco Mundial & 2.47 & 2.50 & 2.47 & 2.45 & 2.48 & 2.52 & 2.57 & 2.60 & 2.74 & 3.01 & 3.13 & 3.15 & 3.36 \\
\hline Diferencia & 0.26 & 0.19 & 0.16 & 0.12 & 0.10 & 0.05 & 0.03 & -0.20 & -0.32 & -0.31 & -0.25 & -0.36 & -0.61 \\
\hline
\end{tabular}

Fuente: Elaboración propia en base a datos de PWT v8.0 y Banco Mundial

Teniendo en cuentas las diferencias que hay entre los tipos de cambio estimados de Bs. a PPP, en la figura 6 se convirtieron las líneas de pobreza estimadas y oficiales a PPP. Lo que se observa en los gráficos es que la línea de pobreza extrema del área rural es la más próxima a la línea internacional de pobreza. Esto implica que si se usa la línea de pobreza extrema internacional se estarían aplicando los criterios de pobreza rural a todo el país. 
Figura 6: Diferencias entre las líneas de indigencia internacional, estimadas y oficiales

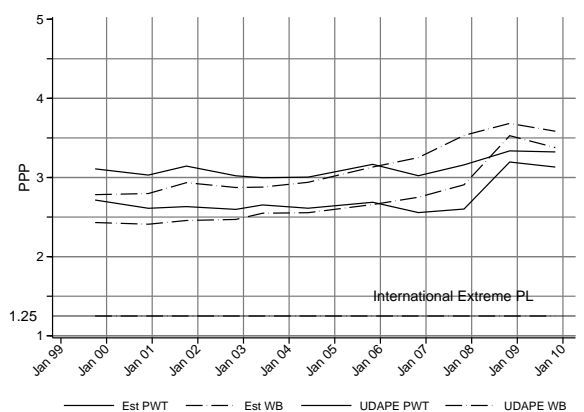

(a) La Paz

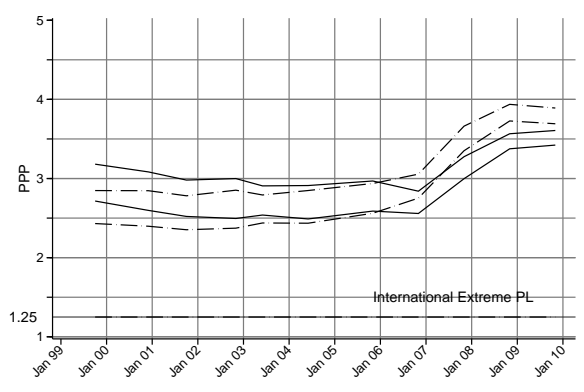

(c) Santa Cruz

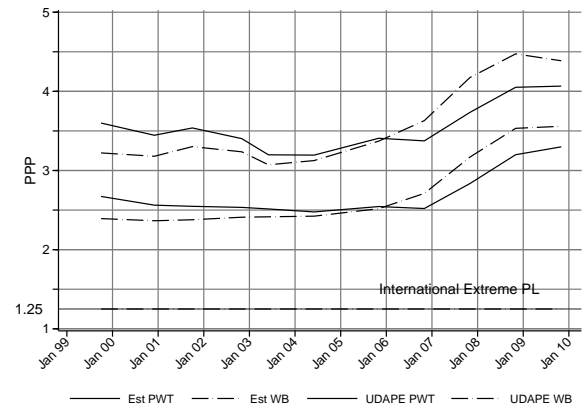

(b) Cochabamba

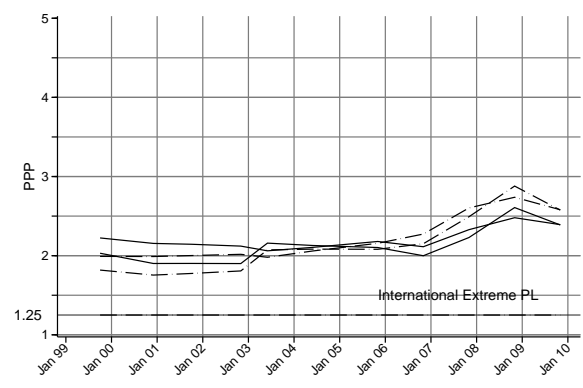

(d) Área rural

Fuente: Elaboración propia con base en la ECH 2003-2004 (INE), UDAPE, PWT v8.0, ICP (Banco Mundial)

\section{Líneas de pobreza}

\subsection{Costo de las necesidades básicas no alimentarias}

El método del costo de las necesidades básicas consiste en establecer un mínimo de consumo adecuado para garantizar las necesidades básicas, y luego establecer el costo monetario que representa alcanzar dichas necesidades. Se considera también como el costo de un nivel de utilidad o un mínimo social normativo para evitar la pobreza (Ravallion, 2000). 
En la práctica se elige una combinación de consumo relacionado con las preferencias del consumidor, de manera que una persona se considera pobre si presenta un nivel de gasto (o ingreso) menor al costo de las necesidades básicas. El componente alimentario se refiere a la estructura y al costo de las CBA construidas previamente, que permiten cubrir los requerimientos promedio de la población según los hábitos de consumo alimentario a los precios vigentes en el mercado.

Para el componente no alimentario serequiere calcular el coeficiente de Orshansky, que se define como el inverso de la fracción de gasto alimentario respecto del gasto total. Dicho coeficiente se multiplica por el costo de la CBA (o línea de indigencia). Frecuentemente el coeficiente de Orschansky se calcula sobre la misma población de referencia que dio lugar a la estructura de la CBA. Por lo tanto, las líneas de pobreza dependen del costo de la CBAy del coeficiente de Engel, supuesto que es justificado en el cálculo de las líneas de pobreza por Wodon (1997), World Bank (1998), CEPAL (1999), Ravallion (1997) entre otros.

Cuadro 11

Coeficiente de Engel y Orshansky

\begin{tabular}{lll}
\hline Dominio & Engel & Orschansky \\
\hline Resto urbano & 0.55 & 1.81 \\
La Paz - El Alto & 0.52 & 1.91 \\
Cochabamba & 0.51 & 1.96 \\
Santa Cruz & 0.49 & 2.05 \\
Área Rural & 0.57 & 1.74 \\
\hline
\end{tabular}

Fuente: Elaboración propia a partir de datos de la ECH 2003-2004 (INE)

Se estimó el inverso de la proporción de gasto alimentario total respecto al gasto total de la población de referencia correspondiente (véase cuadro 11), y éstese multiplicó por el costo de las distintas CBA. En la figura 7 se muestra la evolución de la línea de pobreza, y en el cuadro 12 se documentan los valores de dicha línea a diciembre.

Una característica importante es que la línea de pobreza de Santa Cruz evoluciona de forma cercana a la línea de pobreza de La Paz hasta mediados de 2007. Después de 2007 la línea de pobreza de Santa Cruz se dispara y se crea una brecha en relación a La Paz. De manera similar a las líneas de pobreza extrema, la ciudad de Cochabamba tiene la línea de pobreza más alta en relación a los otros dominios. 
Figura 7: Evolución de la línea de pobreza según dominio (en Bs.)

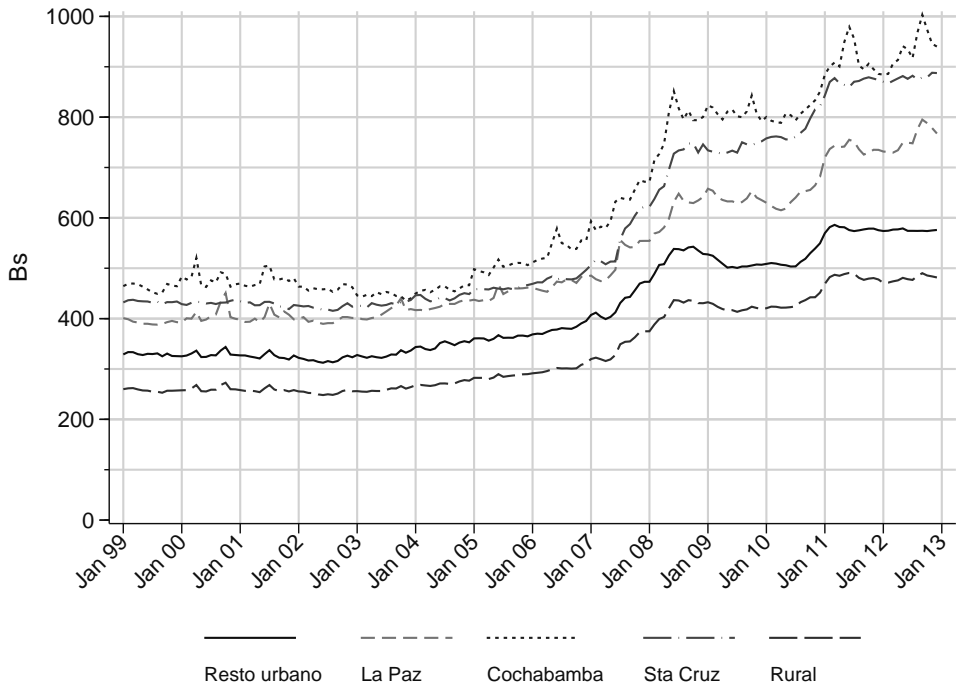

Fuente: Elaboración propia con base en la ECH 2003-2004 (INE)

Cuadro 12

Línea de pobreza a diciembre según dominio (en Bs. por persona al mes)

\begin{tabular}{|l|rrrrr|}
\hline Fecha & Resto Urbano & La Paz & Cochabamba & Santa Cruz & Rural \\
\hline Dic 99 & 325.51 & 392.98 & 464.25 & 434.00 & 257.27 \\
Dic 00 & 327.79 & 399.68 & 468.14 & 437.60 & 259.62 \\
Dic 01 & 326.81 & 398.48 & 480.11 & 426.98 & 258.09 \\
Dic 02 & 323.31 & 401.30 & 462.38 & 423.77 & 255.82 \\
Dic 03 & 336.57 & 418.93 & 440.15 & 436.91 & 264.03 \\
Dic 04 & 352.89 & 435.78 & 466.41 & 448.69 & 276.83 \\
Dic 05 & 364.85 & 461.60 & 506.23 & 466.71 & 289.77 \\
Dic 06 & 395.28 & 482.70 & 554.44 & 496.56 & 311.52 \\
Dic 07 & 473.09 & 554.36 & 671.74 & 622.11 & 374.87 \\
Dic 08 & 528.36 & 641.48 & 800.39 & 745.88 & 430.57 \\
Dic 09 & 506.99 & 635.34 & 792.16 & 751.88 & 419.35 \\
Dic 10 & 549.45 & 681.47 & 850.52 & 821.19 & 451.53 \\
Dic 11 & 575.53 & 735.05 & 885.81 & 873.89 & 477.68 \\
Dic 12 & 576.01 & 767.51 & 940.10 & 887.52 & 481.70 \\
\hline
\end{tabular}

Fuente: Elaboración propia con base en la ECH 2003 - 2004 (INE)

\subsection{Comparando las líneas de pobreza moderada}

En la figura 8 se muestran las diferencias entre las líneas de pobreza estimadas y las líneas oficiales reportadas por UDAPE. Si bien la aplicación del coeficiente de Orshansky 
no cambió las tendencias observadas de las líneas de pobreza, las diferencias entre las líneas estimadas y las oficiales se ampliaron en relación a las líneas de pobreza extrema. En Cochabamba, por ejemplo, la diferencia entre las líneas es de Bs. 143 en 2009. Por otra parte, la diferencia entre las líneas del área rural disminuyó, llegando incluso a ser negativa en 2009 (Bs.3, aproximadamente).

En la figura 9 comparamos las líneas de pobreza estimadas y las oficiales expresadas en PPP, con la línea internacional de pobreza (\$US 2 en PPP). En relación a los dominios definidos, se tiene que la línea de pobreza internacional ofrecería sub-estimaciones de las medidas de pobreza en el área urbana. Sin embargo, ésta es una conjetura que deberá ser revelada a la luz de los datos, pues no solamente importan los niveles de pobreza, sino también su evolución.

Figura 8: Diferencias entre las líneas de indigencia internacional, estimadas y oficiales.

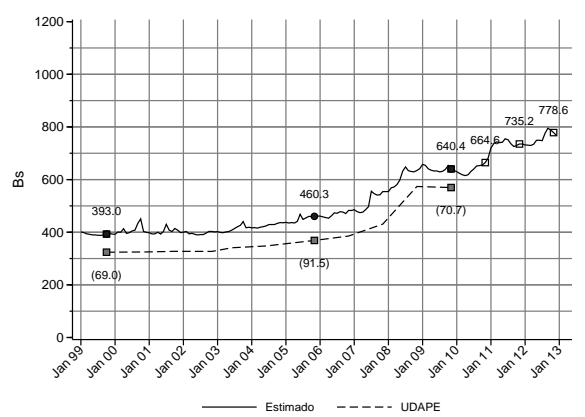

(a) La Paz

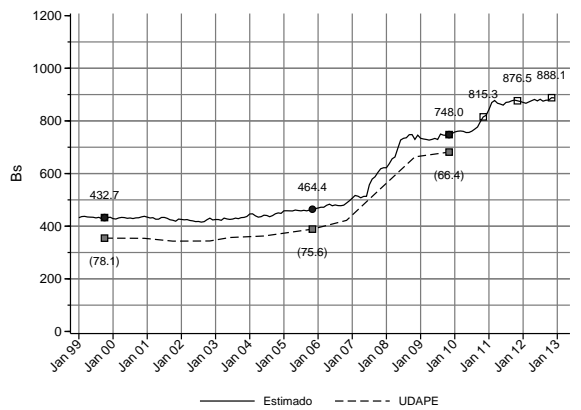

(c) Santa Cruz

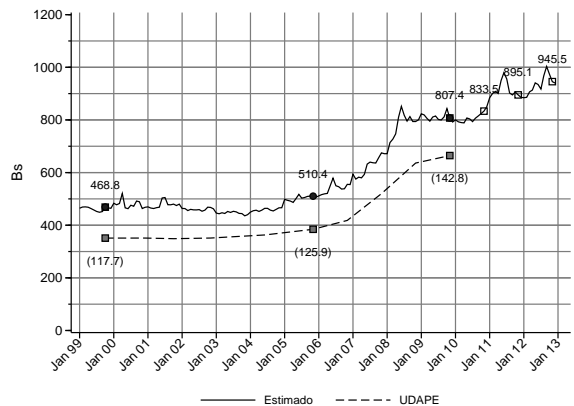

(b) Cochabamba

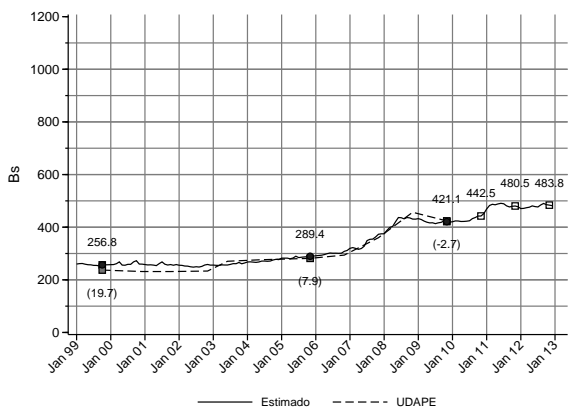

(d) Área rural

Fuente: Elaboración propia con base en la ECH 2003-2004 (INE), UDAPE, PWT v8.0, ICP (Banco Mundial) 


\section{Figura 9: Diferencias entre las líneas de indigencia internacional, estimadas y oficiales.}

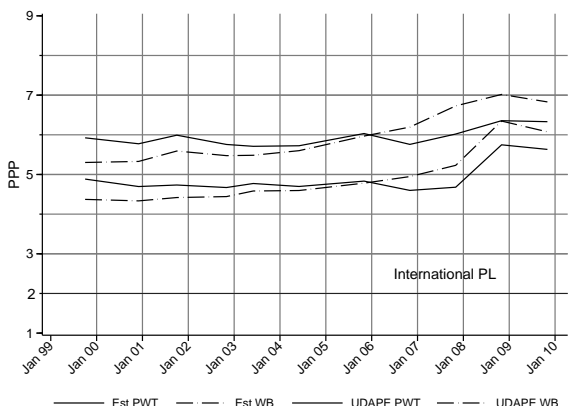

(a) La Paz

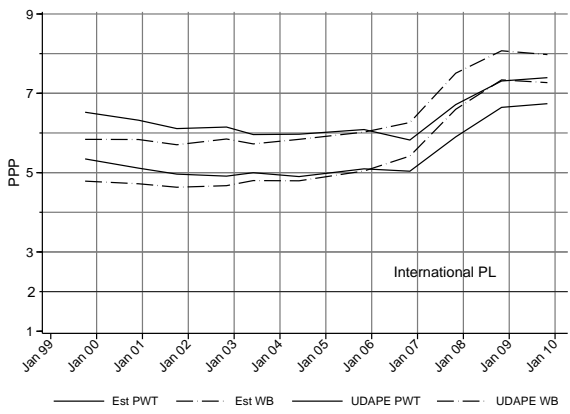

(c) Santa Cruz

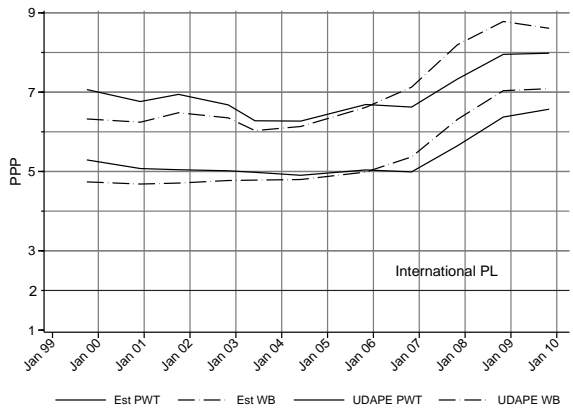

(b) Cochabamba

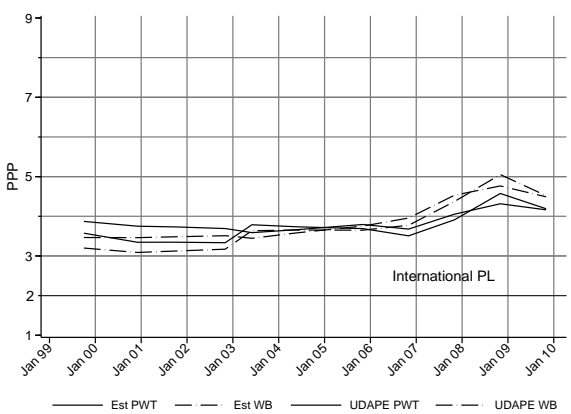

(d) Área rural

Fuente: Elaboración propia con base en la ECH 2003 - 2004 (INE), UDAPE, PWT v8.0, ICP (BancoMundial)

\section{Conclusiones}

Establecer las líneas de pobreza es a menudo la tarea más difícil al momento de medir la pobreza monetaria. Los métodos usados para establecer las líneas de pobreza y medirla tienen diversas implicaciones, tales como qué zona debe tener mayor prioridad, i.e., zona occidental u oriental, al momento de diseñar políticas de reducción de la pobreza. Por otra parte, las líneas de pobreza establecen "cortes" en la distribución de ingresos y permite evidenciar si realmente los pobres se benefician del crecimiento económico. Asimismo, al determinar las líneas de pobreza se puede tener una mejor idea de qué políticas son más efectivas y su robustez para reducir la pobreza. 
Usando la metodología del costo de necesidades básicas en este trabajo se estimaron líneas de pobreza extrema y moderada para cinco dominios. Los resultados nos muestran diferencias significativas entre los umbrales de pobreza establecidos para los dominios, los cuales son fruto de los patrones de consumo y la diferencia entre los precios que enfrenta cada dominio. Al apoyar las estimaciones en los patrones de consumo, se toman en cuenta los productos más representativos que normalmente consumen los hogares de referencia, y al tomar en cuenta los precios del IPC se puede obtener una buena aproximación de la evolución del costo de la canasta básica alimentaria. No obstante, este último detalle implica que se debe tener cuidado al momento de establecer la canasta del IPC, pues implícitamentese delimitan los productos representativos de la canasta básica de alimentos.

Como menciona Ravallion (1992), "La razón más importante para medir la pobreza probablemente no sea la necesidad de un simple número para algún lugar o alguna fecha, sino más bien para hacer comparaciones de pobreza". Ante la variedad de metodologías para establecer las líneas de pobreza, con sus fortalezas y debilidades, serán estas estimaciones las que permitan determinar la robustez de una política para combatir la pobreza. Por ende, lo mejor que se puede hacer es obtener diferentes estimaciones de las medidas de pobreza y tomar decisiones de política a la luz de los datos.

Diferentes instituciones han medido la pobreza bajo el precepto de "one dollar a day", haciendo referencia a las metas del milenio, por ejemplo. No obstante, al proceder de esta manera se deja de lado la realidad propia de la evolución de los precios y patrones de consumo al interior de cada país. Las comparaciones entre las líneas de pobreza oficiales, estimadas y la internacional nos muestran que esta última estaría más cercana a la línea de pobreza rural de Bolivia. Esto implica que, si se usan las líneas de pobreza internacional para hacer perfiles de pobreza, se imponen criterios de pobreza rural sobre el área urbana. Los resultados obtenidos no contemplarían el hecho principal de que las condiciones de pobreza en el área urbana y en el área rural difieren.

Asimismo, las distintas fuentes que estiman el tipo de cambio de Bs. a PPP usan metodologías diferentes, llevando a discrepancias entre sus resultados. Las diferencias introducen una fuente de diferencias en las medidas de pobreza ajenas a la evolución de la pobreza en sí misma. Este resultado es preocupante pues, ceteris paribus, las estimaciones de pobreza usando la línea de pobreza internacional y el tipo de cambio a PPP del PWT mostrarían un mayor número de pobres en relación a los PPP del Banco Mundial en el periodo posteriora 2005. Como se menciona en Deaton (2001), aunque la línea de pobreza 
internacional es una herramienta importante, su dependencia del tipo de cambio a PPP tiene una serie de consecuencias desafortunadas.

La administración actual en Bolivia se ha planteado objetivos específicos de reducción de la pobreza hacia 2025. Dados los insumos oficiales con los que se cuenta para monitorear la evolución de la pobreza, es posible que los resultados encontrados tengan sesgos importantes que lleven a conclusiones erróneas en el monitoreo de la evolución de la pobreza. Visto de otra forma, la evaluación de las políticas sociales y su efecto sobre la reducción de la pobreza dependen en gran medida de los umbrales que las líneas de pobreza monetaria establezcan.

Sin embargo, el monitoreo de la pobreza también depende de la calidad de los datos que se recolectan con las encuestas de hogares. Éste es un detalle de suma importancia al cual muchas veces no sele da la suficiente atención. El tratamiento apropiado de la información tiene un gran efecto sobre los resultados a obtenerse en la investigación. Entonces uno debe preguntarse: ¿Tenemos la información apropiada para monitorear la pobreza en Bolivia?

Finalmente, como numerosas investigaciones han recalcado, la pobreza no es un fenómeno monetario solamente, sino más bien multidimensional. Si bien es posible otorgar recursos a las familias para que éstas mejoren su situación económica, esto no implica que su bienestar aumente. Los recursos monetarios dejan de ser importantes en la medida en que no existan las oportunidades, por ejemplo, de acceso a educación o de conseguir un servicio médico de calidad.

Artículo recibido: 26 de junio de 2013 Aceptado: 27 de septiembre de 2013 


\section{Referencias}

1. Albarracín,J.y Villegas, R. (1997). “Tabla compilada de necesidades medias diarias de energía y nutrientes para la población boliviana”. Ministerio de Salud. La Paz, Bolivia.

2. Bengoa et al. (1989). Archivos Latinoamericanos de Nutrición.

3. Bolivia. Ministerio de Salud y Deportes (2005). "Tabla boliviana de composición química de alimentos”. Serie documentos técnicos, La Paz, Bolivia, cuarta edición.

4. CEPAL (1991). "Magnitud de la pobreza en América Latina en los años ochenta". Comisión Económica para América Latina y El Caribe. Santiago de Chile.

5. ------- (2006). "Panorama social de América latina 2006." Comisión Económica para América Latina y el Caribe. Santiago de Chile

6. - ------- (2006). ICP. Consumo de los hogares: primeros resultados para América del Sur. Programa de Comparación Internacional. Comisión Económica para América Latina y el Caribe. Documento electrónico.

7. ------- (2007). "Principios y aplicación de las nuevas necesidades de energía según el Comité de Expertos FAO/OMS 2004. Serie Estudios estadísticos prospectivos. División de Estadísticas y Proyecciones. Comisión Económica para América Latina y el Caribe. Santiago de Chile.

8. Dandekar, Vinayak, Dandekar, Vishnu y Rath, N. (1971). Poverty in India. Pune, Indian School of Political Economy.

9. Deaton, A. (2001). "Counting the World's Poor: Problems and Possible Solutions". The World Bank Research Observer. 16 (2), 125-147.

10. ------- (2003). Measurement Poverty in a Growing World (or Measurement Growth in a Poor World). Reasearch Program in Development Studies. Woodrow Wilson School. Princeton University.

11. Deaton, A. y Zaidi, S. (s/f). "Directrices para la construcción de agregados de consumo". Banco Mundial. Proyecto de mejoramiento de la medición de las condiciones de vida (MECOVI).

12. Espejo Solá J.(1981).Dietoterapia de lasenfermedades del adulto.Buenos Aires, Argentina, quinta edición.

13. FAO-WHO-UNU (1985). "Necesidades de energía y proteína. Informe de una reunión consultiva conjunta FAO-OMS-UNU de expertos” Organización Mundial de la Salud. Serie de informes técnicos. OMS, Ginebra. 
14. ------ (2001). "Expert Consultation Report on Human Energy Requirements" Interim Report.

15. Feenstra, R. y Diewert, E. (2001). "Imputation and Price Indexes: Theory and Evidence from the International Price Program". U.S. Department of Labor. Bureau of Labor Statistics, Working Paper № 335.

16. Greer, J. y Thorbecke, E. (1986). “A Methodology for Measuring Food Poverty Applied to Kenya”. Journal of Development Economics, № 24, pp.59-74.

17. Hernani-Limarino, W. y Eid, A . (2013). "Unraveling Declining Income Inequality in Bolivia: Do Government Transfers Matter?” Paper Prepared for the IARIW-IBGE Conference on Income, Wealth and Well-Being in Latin America.

18. Herrera, J. (2001). "Food requirements and deficits, Peru 1997-2000 (discussion draft)" IRD- Instituto Nacional de Estadística e Informática (INEI). Fourth Meeting of the Expert Group on Poverty Statistics (Rio Group).

19. Instituto Nacional de Estadística (1992). “Encuesta de presupuestos familiares 1990”. Ministerio de Planeamiento y Coordinación. La Paz, Bolivia.

20. ------- (1993a). "Encuesta de seguimiento al consumo de alimentos. Tabla de equivalencias de pesos de los alimentos crudos en unidades de medida casera al sistema internacional". La Paz, Bolivia.

21. - ----- (1993b). "Encuesta de seguimiento al consumo de alimentos. Tabla de composición química de alimentos crudos”. La Paz, Bolivia.

22. - ------ (1994). "Alimentación y nutrición (ciudades de La Paz, Cochabamba, Santa Cruz y El Alto). Un análisis a partir de la encuesta de presupuestos familiares”. La Paz, Bolivia.

23. - -----(2005)."Encuesta continua de hogares 2003-2004. Metodología y Resultados”. La Paz, Bolivia.

24. Kakwani, N. y Pernia, E. (2000). “What is Pro-Poor Growth?” Asian Development Review, 18 (1), 1-16. Manila: Asian Development Bank.

25. Kakwani, N. y Son, Hyun (2006). "New Global Poverty Counts". Working Paper No 29, International Poverty Centre. United Nations for Development Program. Brasilia.

26. Medina, Fy Galván, M. (2006). “Imputación de datos: teoría y práctica”. Serie Estudios estadísticos prospectivos. Comisión Económica Para América Latina. Santiago de Chile. 
27. Morales, R.(1983). "Medición de las variaciones del poder de compra asociadas a las variaciones de los precios y metodología para el cálculo del ingreso mínimo vital”. Centro de Investigación y Consultoría. CINCO. La Paz, Bolivia.

28. Osmani, S. (1982). Economic Inequality and Group Welfare. Oxford: Oxford University Press.

29. Paul, S. (1989). "A Model of Constructing the Poverty Line". Journal of Development Economics, No 30, pp. 129-144.

30. Ravallion, M. (2000). "Las líneas de pobreza en la teoría y en la práctica. Banco Mundial". Proyecto de mejoramiento de la medición de las condiciones de vida (MECOVI).

31. Ravallion, M., Datt, G. y Van den Walle, D. (1991). "Quantifying absolute poverty in the developing world”. Review of Income of Wealth, No 37, pp. 345-361.

32. Ravallion, M. y Bidani, B. (1994). "How Robust is a Poverty Profile". World Bank Economic Review, No, 8, pp. 75-102

33. Ravallion, M. y Chen, S. (1997). "What can Survey Data tell us About Recent Challenges in Distribution and Poverty”. World Bank Economic Review, No 11, pp. 357-382.

34. Ravallion, M., Chen, S. y Sangraula, P. (2008). "Dolar a day revisited". Policy Research Working Paper, 4620, Washington D.C. World Bank.

35. Rio Group (2006). Compendium of Best Practices in Poverty Measurement. Expert Group on Poverty Statistics. Rio de Janeiro.

36. Villegas, R. (2005). "Alimentos crudos. Factores de corrección y equivalencias de pesos y medidas. Instrumentos para el análisis agroalimentario nutricional y dietético". Ministerio de Salud y Deportes. La Paz, Bolivia, segunda edición.

37. Wodon, Quentin (1997)."Food Energy Intake and Cost of Basic Needs: Measuring Poverty in Bangladesh". Journal of Development Studies. 34 (2), 66-101. London.

38. UDAPSO (1995). "Metodología para la construcción de la canasta básica de alimentos". Cuadernos de desarrollo humano No 2. Unidad de Análisis de Políticas Sociales (UDAPSO), Instituto Nacional de Alimentación y Nutrición (INAN) y asistencia técnica de la Comisión Económica para América Latina y el Caribe (CEPAL), La Paz. 


\section{Anexos}

Cuadro 13

La Paz: canasta básica de alimentos (en términos per cápita al día)

\begin{tabular}{|c|c|c|c|c|}
\hline Artículo & Cantidad & Caloría & Proteína & Grasas \\
\hline Pan de batalla & 118.69 & 350.87 & 10.56 & 1.05 \\
\hline Arroz & 64.81 & 236.07 & 4.85 & 0.31 \\
\hline Harina de trigo & 14.40 & 53.04 & 1.71 & 0.23 \\
\hline Fideo para guisar & 43.16 & 151.53 & 4.87 & 0.54 \\
\hline Carne con hueso de res & 22.60 & 48.93 & 6.53 & 2.18 \\
\hline Pulpa & 27.47 & 34.17 & 4.81 & 0.74 \\
\hline Carne de pollo entero & 22.29 & 34.92 & 3.51 & 2.14 \\
\hline Pejerrey & 2.30 & 2.18 & 0.45 & 0.02 \\
\hline Karachi & 2.50 & 3.41 & 0.50 & 0.10 \\
\hline Sardina & 1.09 & 2.55 & 0.29 & 0.16 \\
\hline Aceite de girasol & 15.83 & 140.45 & 0.00 & 15.76 \\
\hline Margarina & 1.13 & 8.10 & 0.01 & 0.90 \\
\hline Leche pasteurizada & 20.57 & 11.02 & 0.64 & 0.55 \\
\hline Leche en polvo & 1.50 & 6.70 & 0.33 & 0.32 \\
\hline Queso criollo & 5.03 & 18.36 & 1.39 & 1.28 \\
\hline Huevo de gallina & 11.66 & 16.62 & 1.32 & 1.08 \\
\hline Zanahoria & 31.95 & 14.53 & 0.43 & 0.08 \\
\hline Tomate & 25.14 & 5.86 & 0.28 & 0.09 \\
\hline Zapallo/iscariote & 7.33 & 3.01 & 0.09 & 0.02 \\
\hline Lechuga & 3.08 & 0.55 & 0.05 & 0.01 \\
\hline Cebolla entera, cebolla verde, cebolla con cola & 27.91 & 8.05 & 0.56 & 0.17 \\
\hline Cebolla cortada, solo cabeza, cebolla cabeza & 21.69 & 11.07 & 0.37 & 0.05 \\
\hline Papa & 119.79 & 145.20 & 3.13 & 0.17 \\
\hline Chuño & 7.33 & 23.90 & 0.24 & 0.02 \\
\hline Arveja & 5.56 & 4.67 & 0.39 & 0.02 \\
\hline Haba & 4.38 & 4.93 & 0.54 & 0.02 \\
\hline Maní crudo & 0.49 & 3.09 & 0.14 & 0.25 \\
\hline Mandarina & 16.63 & 11.70 & 0.14 & 0.05 \\
\hline Naranja & 5.30 & 2.52 & 0.04 & 0.01 \\
\hline Manzana & 5.71 & 3.76 & 0.02 & 0.01 \\
\hline Papaya & 11.13 & 5.12 & 0.05 & 0.01 \\
\hline Plátano/guineo/banano & 49.24 & 42.45 & 0.50 & 0.34 \\
\hline Plátano/postre para cocinar & 5.55 & 9.16 & 0.07 & 0.02 \\
\hline Azúcar granulada & 39.00 & 155.64 & 0.00 & 0.00 \\
\hline Mermeladas / compotas & 0.58 & 1.71 & 0.01 & 0.00 \\
\hline Helados & 2.88 & 4.18 & 0.16 & 0.07 \\
\hline Café tostado y molido & 2.60 & 1.25 & 0.02 & 0.00 \\
\hline Café instantáneo & 0.06 & 0.11 & 0.00 & 0.00 \\
\hline Té en bolsita & 0.46 & 0.34 & 0.00 & 0.00 \\
\hline Cacao/cocoa en polvo con azúcar u otro edulcorante & 0.82 & 5.40 & 0.32 & 0.20 \\
\hline Mayonesa & 0.42 & 5.69 & 0.08 & 0.28 \\
\hline Sal & 7.11 & 0.00 & 0.00 & 0.00 \\
\hline Caldos en cubitos/ajinomoto/zasonadores & 0.43 & 1.29 & 0.12 & 0.00 \\
\hline Bebidas gaseosas & 93.07 & 40.97 & 0.00 & \\
\hline Jugos de fruta & 5.17 & 2.43 & 0.01 & \\
\hline Cerveza blanca & 3.95 & 3.17 & 0.04 & \\
\hline Almuerzo en local & 101.47 & 164.15 & 7.84 & 5.38 \\
\hline Almuerzo en mercado o puesto & 48.34 & 89.93 & 4.00 & 3.38 \\
\hline Cena en local & 15.90 & 32.16 & 1.06 & 1.16 \\
\hline Cena en mercado o puesto & 6.93 & 55.36 & 0.33 & 0.08 \\
\hline Desayuno/te en mercado/puesto & 16.03 & 81.54 & 2.61 & 1.87 \\
\hline Frescos hervidos no alcohólicos & 19.33 & 38.85 & 0.30 & 0.09 \\
\hline Jugo de fruta & 21.59 & 16.31 & 0.18 & 0.18 \\
\hline Cerveza consumida fuera del hogar & 9.29 & 4.04 & 0.02 & 0.00 \\
\hline
\end{tabular}

Fuente: Elaboración propia a partir de datos del INE 
Cuadro 14

Cochabamba: Canasta Básica de Alimentos (en términos per-cápita al día)

\begin{tabular}{|c|c|c|c|c|}
\hline Artículo & Cantidad & Caloría & Proteína & Grasas \\
\hline Pan de batalla & 146.54 & 437.10 & 13.14 & 1.50 \\
\hline Arroz & 41.58 & 152.80 & 3.14 & 0.23 \\
\hline Fideo para guisar & 48.03 & 170.15 & 5.46 & 0.69 \\
\hline Carne con hueso de res & 44.83 & 103.92 & 13.71 & 4.88 \\
\hline Pulpa & 6.05 & 8.06 & 1.12 & 0.18 \\
\hline Carne de pollo entero & 21.88 & 36.69 & 3.65 & 2.37 \\
\hline Sábalo & 0.91 & 1.59 & 0.20 & 0.07 \\
\hline Aceite de girasol & 17.10 & 151.58 & . & 16.94 \\
\hline Leche natural & 14.82 & 9.03 & 0.52 & 0.44 \\
\hline Leche pasteurizada & 38.09 & 22.44 & 1.35 & 1.14 \\
\hline Leche en polvo & 2.85 & 14.04 & 0.72 & 0.69 \\
\hline Leche saborizada & 5.01 & 2.30 & 0.11 & 0.06 \\
\hline Queso criollo & 3.51 & 14.10 & 1.10 & 1.00 \\
\hline Huevo de gallina & 10.58 & 16.58 & 1.36 & 1.10 \\
\hline Zanahoria & 37.49 & 16.55 & 0.50 & 0.12 \\
\hline Locoto & 6.30 & 2.04 & 0.08 & 0.01 \\
\hline Tomate & 54.83 & 12.39 & 0.59 & 0.23 \\
\hline Cebolla entera, cebolla verde, cebolla con cola & 14.39 & 4.03 & 0.29 & 0.11 \\
\hline Cebolla cortada, solo cabeza, cebolla cabeza & 48.59 & 24.06 & 0.82 & 0.14 \\
\hline Vainita & 6.22 & 3.08 & 0.14 & 0.02 \\
\hline Рара & 138.35 & 172.81 & 3.72 & 0.18 \\
\hline Arveja & 6.08 & 5.44 & 0.42 & 0.11 \\
\hline Haba & 8.00 & 9.58 & 0.98 & 0.18 \\
\hline Durazno & 4.87 & 3.62 & 0.03 & 0.01 \\
\hline Mandarina & 11.11 & 8.04 & 0.09 & 0.04 \\
\hline Naranja & 7.14 & 3.49 & 0.05 & 0.02 \\
\hline Manzana & 4.45 & 3.01 & 0.01 & 0.01 \\
\hline Papaya & 10.25 & 4.85 & 0.05 & 0.01 \\
\hline Plátano/guineo/banano & 79.38 & 70.36 & 0.81 & 0.60 \\
\hline Azúcar granulada & 28.31 & 112.97 & 0.00 & 0.00 \\
\hline Caramelos/dulces/tofees & 0.57 & 2.23 & 0.00 & 0.00 \\
\hline Helados & 5.27 & 7.64 & 0.27 & 0.09 \\
\hline Café instantáneo & 0.23 & 0.19 & 0.00 & 0.00 \\
\hline Cacao/cocoa en polvo con azúcar u otro edulcorante & 1.67 & 4.78 & 0.28 & 0.12 \\
\hline Mayonesa & 6.79 & 6.94 & 0.19 & 0.21 \\
\hline Bebidas gaseosas & 88.07 & 36.81 & 0.00 & \\
\hline Jugos de fruta & 6.92 & 3.09 & 0.01 & . \\
\hline Cerveza blanca & 4.39 & 2.86 & 0.02 & . \\
\hline Almuerzo en local & 64.82 & 109.62 & 6.74 & 3.61 \\
\hline Almuerzo en mercado o puesto & 49.96 & 97.16 & 5.56 & 3.67 \\
\hline Cena en local & 12.12 & 25.62 & 1.09 & 0.93 \\
\hline Cena en mercado o puesto & 5.31 & 44.38 & 0.34 & 0.06 \\
\hline Plato especial en local & 13.03 & 28.92 & 1.19 & 1.09 \\
\hline Plato especial en mercado o puesto & 12.57 & 24.45 & 1.21 & 0.99 \\
\hline Sándwich & 4.79 & 16.35 & 0.88 & 0.31 \\
\hline Chicha alcohólica consumida fuera del hogar & 94.20 & 115.28 & 1.34 & 0.57 \\
\hline
\end{tabular}

Fuente: Elaboración propia a partir de datos del INE 
Cuadro 15

Santa Cruz: Canasta Básica de Alimentos (en términos per-cápita al día)

\begin{tabular}{|c|c|c|c|c|}
\hline Artículo & Cantidad & Caloría & Proteína & Grasas \\
\hline Pan de batalla & 236.96 & 701.71 & 21.14 & 2.08 \\
\hline Arroz & 68.44 & 249.71 & 5.14 & 0.33 \\
\hline Fideo para guisar & 23.30 & 81.94 & 2.64 & 0.29 \\
\hline Carne con hueso de res & 20.90 & 52.11 & 6.79 & 2.49 \\
\hline Hueso/ cabeza de res & 11.16 & 0.00 & 0.00 & 0.00 \\
\hline Pulpa & 17.34 & 24.84 & 3.42 & 0.58 \\
\hline Carne de pollo entero & 20.00 & 36.08 & 3.55 & 2.37 \\
\hline Sábalo & 0.35 & 0.67 & 0.08 & 0.03 \\
\hline Sardina & 0.72 & 1.63 & 0.18 & 0.10 \\
\hline Aceite de girasol & 11.89 & 106.53 & 0.00 & 11.87 \\
\hline Mantequilla & 0.45 & 3.16 & 0.01 & 0.34 \\
\hline Margarina & 0.54 & 3.93 & 0.00 & 0.44 \\
\hline Leche natural & 25.47 & 15.36 & 0.88 & 0.75 \\
\hline Leche pasteurizada & 23.44 & 13.67 & 0.82 & 0.69 \\
\hline Leche en polvo & 0.41 & 2.02 & 0.10 & 0.10 \\
\hline Queso criollo & 3.20 & 12.75 & 0.99 & 0.90 \\
\hline Huevo de gallina & 6.81 & 10.58 & 0.86 & 0.70 \\
\hline Zanahoria & 24.98 & 14.50 & 0.39 & 0.06 \\
\hline Tomate & 51.31 & 15.25 & 0.66 & 0.15 \\
\hline Lechuga & 3.45 & 0.78 & 0.07 & 0.01 \\
\hline Papa & 52.82 & 65.40 & 1.43 & 0.07 \\
\hline Yuca/mandioca & 7.36 & 10.79 & 0.08 & 0.02 \\
\hline Arveja & 2.22 & 3.79 & 0.24 & 0.11 \\
\hline Haba & 1.12 & 2.55 & 0.21 & 0.07 \\
\hline Mandarina & 9.41 & 5.85 & 0.07 & 0.03 \\
\hline Naranja & 3.96 & 1.66 & 0.03 & 0.01 \\
\hline Manzana & 1.19 & 0.69 & 0.00 & 0.00 \\
\hline Plátano/guineo/banano & 15.02 & 11.44 & 0.14 & 0.09 \\
\hline Plátano/postre para cocinar & 12.50 & 18.21 & 0.14 & 0.04 \\
\hline Azúcar granulada & 38.79 & 153.01 & & \\
\hline Café tostado y molido & 2.38 & 1.29 & 0.03 & 0.00 \\
\hline Café instantáneo & 0.14 & 0.30 & 0.00 & 0.00 \\
\hline Té en bolsita & 0.48 & 0.40 & 0.01 & 0.00 \\
\hline Cacao/cocoa en polvo con azúcar u otro edulcorante & 0.29 & 2.16 & 0.18 & 0.10 \\
\hline Sal & 7.34 & 0.00 & 0.00 & 0.00 \\
\hline Caldos en cubitos/ajinomoto/zasonadores & 0.47 & 6.30 & 0.11 & 0.28 \\
\hline Bebidas gaseosas & 19.83 & 8.52 & 0.00 & \\
\hline Jugos de fruta & 3.87 & 1.78 & 0.01 & \\
\hline Polvos para preparar refrescos o jugos & 0.75 & 2.95 & 0.00 & \\
\hline Cerveza blanca & 0.43 & 0.30 & 0.01 & \\
\hline Almuerzo en local & 130.81 & 218.80 & 9.88 & 7.23 \\
\hline Almuerzo en mercado o puesto & 27.81 & 53.49 & 2.25 & 2.02 \\
\hline Cena en local & 36.08 & 75.44 & 2.36 & 2.74 \\
\hline Cena en mercado o puesto & 4.38 & 36.16 & 0.20 & 0.05 \\
\hline Desayuno/te en mercado/puesto & 6.64 & 34.91 & 1.06 & 0.81 \\
\hline Chichas no alcohólicas consumida fuera del hogar & 44.71 & 59.60 & 0.69 & 0.66 \\
\hline
\end{tabular}

Fuente: Elaboración propia a partir de datos del INE 
Cuadro 16

Resto Urbano: Canasta Básica de Alimentos (en términos per-cápita al día)

\begin{tabular}{|c|c|c|c|c|}
\hline Artículo & Cantidad & Caloría & Proteína & Grasas \\
\hline Pan de batalla & 160.37 & 473.91 & 14.23 & 1.57 \\
\hline Arroz & 69.37 & 252.56 & 5.18 & 0.37 \\
\hline Harina de trigo & 10.53 & 38.77 & 1.25 & 0.19 \\
\hline Fideo para guisar & 46.20 & 162.14 & 5.20 & 0.64 \\
\hline Carne con hueso de res & 30.71 & 70.35 & 9.44 & 3.28 \\
\hline Carne molida de res & 6.16 & 8.22 & 1.14 & 0.17 \\
\hline Pulpa & 8.96 & 11.78 & 1.67 & 0.27 \\
\hline Carne de pollo entero & 16.56 & 27.44 & 2.78 & 1.76 \\
\hline Sábalo & 0.52 & 0.77 & 0.10 & 0.03 \\
\hline Sardina & 1.93 & 3.36 & 0.43 & 0.16 \\
\hline Aceite de girasol & 19.51 & 172.45 & & 19.28 \\
\hline Leche natural & 10.77 & 6.58 & 0.37 & 0.31 \\
\hline Leche pasteurizada & 5.38 & 3.18 & 0.19 & 0.16 \\
\hline Leche en polvo & 1.12 & 5.54 & 0.28 & 0.26 \\
\hline Queso criollo & 3.60 & 14.50 & 1.11 & 1.00 \\
\hline Huevo de gallina & 9.52 & 14.97 & 1.20 & 0.96 \\
\hline Zanahoria & 37.82 & 17.79 & 0.58 & 0.13 \\
\hline Locoto & 3.31 & 1.14 & 0.05 & 0.00 \\
\hline Tomate & 45.16 & 10.88 & 0.57 & 0.20 \\
\hline Cebolla entera, cebolla verde, cebolla con cola & 14.57 & 4.35 & 0.34 & 0.12 \\
\hline Cebolla cortada, solo cabeza, cebolla cabeza & 43.39 & 22.90 & 0.84 & 0.13 \\
\hline Рара & 153.74 & 194.94 & 4.08 & 0.21 \\
\hline Arveja & 5.60 & 5.00 & 0.41 & 0.02 \\
\hline Haba & 3.44 & 4.10 & 0.44 & 0.02 \\
\hline Maní crudo & 0.79 & 5.27 & 0.24 & 0.39 \\
\hline Mandarina & 9.78 & 6.72 & 0.08 & 0.04 \\
\hline Naranja & 6.27 & 2.91 & 0.04 & 0.02 \\
\hline Manzana & 4.90 & 3.15 & 0.01 & 0.01 \\
\hline Sandía & 2.68 & 0.98 & 0.01 & 0.00 \\
\hline Uva & 1.79 & 1.24 & 0.01 & 0.01 \\
\hline Plátano/guineo/banano & 33.61 & 28.33 & 0.34 & 0.28 \\
\hline Plátano/postre para cocinar & 9.42 & 15.19 & 0.11 & 0.04 \\
\hline Azúcar granulada & 41.36 & 164.99 & 0.00 & 0.00 \\
\hline Helados & 3.46 & 5.02 & 0.20 & 0.10 \\
\hline Café tostado y molido & 2.29 & 1.13 & 0.02 & 0.00 \\
\hline Té en bolsita & 0.61 & 0.46 & 0.01 & 0.00 \\
\hline Cacao/cocoa en polvo con azúcar u otro edulcorante & 0.76 & 5.10 & 0.34 & 0.13 \\
\hline Mayonesa & 0.15 & 5.29 & 0.09 & 0.17 \\
\hline Sal & 12.51 & 0.00 & 0.00 & 0.00 \\
\hline Caldos en cubitos/ajinomoto/zasonadores & 0.15 & 1.17 & 0.13 & 0.00 \\
\hline Bebidas gaseosas & 52.52 & 25.51 & & . \\
\hline Vino tinto o blanco & 0.95 & 1.31 & 0.01 & . \\
\hline Cerveza blanca & 2.47 & 2.65 & 0.04 & \\
\hline Almuerzo en local & 54.73 & 108.63 & 4.38 & 3.25 \\
\hline Almuerzo en mercado o puesto & 33.08 & 75.51 & 2.84 & 2.59 \\
\hline Cena en local & 15.71 & 38.98 & 1.09 & 1.28 \\
\hline Plato especial en mercado o puesto & 11.01 & 25.14 & 0.82 & 0.93 \\
\hline Chicha alcohólica consumida fuera del hogar & 60.62 & 70.72 & 0.86 & 0.61 \\
\hline
\end{tabular}

Fuente: Elaboración propia a partir de datos del INE 
Área rural: Canasta Básica de Alimentos (en términos per-cápita al día)

\begin{tabular}{|c|c|c|c|c|}
\hline Artículo & Cantidad & Caloría & Proteína & Grasas \\
\hline Pan de batalla & 105.18 & 310.10 & 9.38 & 0.80 \\
\hline Arroz & 98.93 & 359.37 & 7.43 & 0.41 \\
\hline Maíz & 25.55 & 92.82 & 2.21 & 1.33 \\
\hline Harina de trigo & 23.47 & 86.20 & 2.80 & 0.32 \\
\hline Fideo para guisar & 44.49 & 155.80 & 5.03 & 0.48 \\
\hline Carne con hueso de res & 21.40 & 47.25 & 7.06 & 1.88 \\
\hline Pulpa & 9.29 & 11.78 & 1.86 & 0.23 \\
\hline Carne de cordero & 5.33 & 12.68 & 0.96 & 0.89 \\
\hline Carne de pollo entero & 9.19 & 14.68 & 1.65 & 0.80 \\
\hline Sábalo & 3.35 & 5.13 & 0.74 & 0.16 \\
\hline Sardina & 2.81 & 5.12 & 0.72 & 0.21 \\
\hline Aceite de girasol & 22.51 & 201.42 & . & 22.41 \\
\hline Leche natural & 14.66 & 8.96 & 0.51 & 0.44 \\
\hline Leche en polvo & 0.79 & 3.91 & 0.20 & 0.19 \\
\hline Queso criollo & 2.80 & 11.28 & 0.87 & 0.80 \\
\hline Huevo de gallina & 6.82 & 10.73 & 0.87 & 0.71 \\
\hline Zanahoria & 32.92 & 22.81 & 0.88 & 0.13 \\
\hline Tomate & 36.24 & 12.86 & 0.79 & 0.19 \\
\hline Cebolla entera, cebolla verde, cebolla con cola & 11.05 & 4.86 & 0.44 & 0.11 \\
\hline Cebolla cortada, solo cabeza, cebolla cabeza & 28.54 & 22.19 & 0.96 & 0.10 \\
\hline Papa & 127.02 & 154.81 & 3.42 & 0.18 \\
\hline Chuño & 13.25 & 43.42 & 0.45 & 0.03 \\
\hline Yuca/mandioca & 28.91 & 41.72 & 0.30 & 0.07 \\
\hline Arveja & 4.36 & 7.66 & 0.48 & 0.04 \\
\hline Haba & 4.80 & 11.28 & 0.93 & 0.06 \\
\hline Mandarina & 4.59 & 3.05 & 0.04 & 0.02 \\
\hline Naranja & 4.13 & 1.85 & 0.03 & 0.01 \\
\hline Manzana & 3.29 & 2.04 & 0.01 & 0.01 \\
\hline Sandía & 2.18 & 0.77 & 0.01 & 0.00 \\
\hline Plátano/guineo/banano & 28.98 & 23.59 & 0.28 & 0.22 \\
\hline Plátano/postre para cocinar & 14.14 & 22.02 & 0.16 & 0.06 \\
\hline Azúcar granulada & 52.06 & 203.09 & & . \\
\hline Café tostado y molido & 5.00 & 10.49 & 0.68 & \\
\hline Sal & 16.31 & 0.00 & 0.00 & 0.00 \\
\hline Caldos en cubitos/ajinomoto/zasonadores & 0.64 & 2.81 & 0.10 & 0.05 \\
\hline Bebidas gaseosas & 31.95 & 16.35 & & . \\
\hline Vino tinto o blanco & 3.61 & 3.19 & 0.05 & . \\
\hline Cerveza blanca & 3.73 & 2.56 & 0.09 & . \\
\hline Almuerzo en local & 35.99 & 66.02 & 5.51 & 2.05 \\
\hline Almuerzo en mercado o puesto & 9.45 & 19.94 & 1.55 & 0.71 \\
\hline Cena en local & 10.16 & 23.30 & 1.35 & 0.80 \\
\hline Plato especial en mercado o puesto & 3.05 & 6.44 & 0.43 & 0.25 \\
\hline Desayuno/te en local & 6.08 & 14.96 & 0.71 & 0.53 \\
\hline Jugo de fruta & 7.95 & 15.60 & 0.17 & 0.18 \\
\hline Chicha alcohólica consumida fuera del hogar & 46.93 & 26.09 & 0.43 & 0.15 \\
\hline
\end{tabular}

Fuente: Elaboración propia a partir de datos del INE 